\title{
Long and short term variability of seven blazars in six near-infrared/optical bands ${ }^{\star}$
}

\author{
A. Sandrinelli ${ }^{1,2}$, S. Covino ${ }^{2}$, and A. Treves ${ }^{1,3}$ \\ 1 Università dell'Insubria, via Valleggio 11, 22100 Como, Italy \\ 2 INAF, Osservatorio Astronomico di Brera, via Bianchi 46, 23807 Merate (LC), Italy \\ e-mail: angela.sandrinelli@brera.inaf.it \\ 3 Affiliated to INAF, INFN, ICRA
}

Received 24 March 2013 / Accepted 19 July 2013

\begin{abstract}
Context. We present the light curves of six BL Lac objects, PKS 0537-441, PKS 0735+17, OJ 287, PKS 2005-489, PKS 2155-304, and W Comae, and of the flat spectrum radio quasar PKS 1510-089, as a part of a photometric monitoring program in the nearinfrared/optical bands started in 2004. All sources are Fermi blazars.

Aims. Our purpose is to investigate flux and spectral variability on short and long time scales. Systematic monitoring, independent of the activity of the source, guarantees large sample size statistics, and allows an unbiased view of different activity states on weekly or daily time scales for the whole timeframe and on nightly time scales for some epochs.

Methods. Data were obtained with the REM telescope located at the ESO premises of La Silla (Chile). Light curves were gathered in the optical/near-infrared VRIJHK bands from April 2005 to June 2012.

Results. Variability $\gtrsim 3$ mag is observed in PKS 0537-441, PKS 1510-089 and PKS 2155-304, the largest ranges spanned in the near-infrared. The color intensity plots show rather different morphologies. The spectral energy distributions in general are well fitted by a power law, with some deviations that are more apparent in low states. Some variability episodes during a night interval are well documented for PKS 0537-441 and PKS 2155-304. For the latter source the variability time scale implies a large relativistic beaming factor.
\end{abstract}

Key words. BL Lacertae objects: general - galaxies: photometry - galaxies: active

\section{Introduction}

Blazars are active galactic nuclei (AGNs) characterized by spectra extending from radio to $\mathrm{GeV}$ and $\mathrm{TeV}$ bands, high variability and polarization. An important subclass is that of BL Lac objects, where contrary to other species of AGNs, emission lines are very weak, if not absent. Ever since the seminal paper of Blandford \& Rees (1978), the basic model of these sources requires nonthermal emission from a relativistic jet, which is pointed in the observer's direction. The thermal emission associated to an accretion disk or to the broad emission line region is only a fraction of that from the jet. The jet emission is most probably dominated by synchrotron radiation of relativistic electrons and by Compton emission through the scattering of electrons off synchrotron photons or thermal ones. This gives rise to the typical "two-peaked shape" of the spectral energy distribution (SED), when studied over a broad energy band. The variability, which is present at all bands, is a basic tool for constraining the model, since it gives information on the size of the emitting region and on the relativistic beaming factor, which transforms the quantities measured in the observer frame into those of the emitting region.

Here we present optical-NIR photometry of seven blazars, therefore we are probing the synchrotron component, where the

* Full Table 3 is only available at the CDS via anonymous ftp to cdsarc.u-strasbg.fr $(130.79 .128 .5)$ or via

http://cdsarc.u-strasbg.fr/viz-bin/qcat?]/A+A/562/A79 first peak of the SED is located. As is apparent from the literature and from our results (see e.g. Impiombato et al. 2011; Bonning et al. 2012, and references therein), the variability pattern of blazars in the optical is rather complex. On time scales of days or months, one can explore modifications in the jet structure or accretion disk, and possibly their interaction. On the other hand, when the time scales are hours, we are close to the scales of the expected radius of the central black hole. General and special relativistic effects become dominant.

Long but sparse exposures have been obtained through the REM telescope, which being robotic is very well fitted for systematic observations of extensive duration. The telescope, the CCD cameras, and the photometry procedures are described in Sect. 2 and the blazar sample in Sect. 3. The variability on time scales larger than one day is presented in Sect. 4 and short term variability in Sect. 5. A discussion of the results is given in Sect. 6.

\section{Telescope, camera and photometric procedures}

The Rapid Eye Mounting Telescope (REM, Zerbi \& Rem Team 2001; Covino et al. 2004) is a robotic telescope located at the ESO Cerro La Silla observatory (Chile). It was built with the main motivation to promptly observe the gamma ray burst detected by the Swift mission. REM has a Ritchey-Chretien configuration with a $60 \mathrm{~cm} f / 2.2$ primary and an overall $f / 8$ focal 
Table 1. Blazar sample.

\begin{tabular}{lcccccc}
\hline \hline Source & $\begin{array}{c}\text { Coordinates } \\
\text { RA Dec } \\
{[\mathrm{h}: \mathrm{m}: \mathrm{s}] \text { [d:m:s] }}\end{array}$ & $\begin{array}{c}\text { Optical } \\
\text { Class }{ }^{(2)}\end{array}$ & $\begin{array}{c}\text { SED } \\
\text { Class }{ }^{(3)}\end{array}$ & Redshift & TeV Source ${ }^{(4)}$ & References \\
\hline PKS 0537-441 & $05: 38: 50.35-44: 05: 09.05$ & BL Lac & LSP & 0.896 & & $(\mathrm{a})$ \\
PKS 0735+17 & $07: 38: 07.39+17: 42: 18.00$ & BL Lac & LSP & 0.424 & & $($ b) \\
OJ 287 & $08: 54.48 .87+20.06: 30.64$ & BL Lac & LSP & 0.3060 & & $(\mathrm{c})$ \\
PKS 1510-089 & $05: 12: 50.53-09: 05: 59.83$ & FSRQ & LSP & 0.3599 & Y & Y \\
PKS 2005-489 & $20: 09: 25.39-48: 49: 53.72$ & BL Lac & HSP & 0.071 & Y & (d) \\
PKS 2155-304 & $21: 58: 52.07-30: 13: 32.12$ & BL Lac & HSP & 0.117 & Y & (e) \\
W Comae & $12: 21: 31.69+28: 13.58 .50$ & BL Lac & ISP & 0.1029 & & \\
\hline
\end{tabular}

Notes. ${ }^{(1)}$ ICRS coordinates (J2000) and redshifts from SIMBAD ${ }^{1}$; (2) Class from Massaro et al. (2012); (3) Classification of the spectral energy distribution: LSP means low synchrotron peaked $\left(v_{\mathrm{S}}<10^{14} \mathrm{~Hz}\right.$, where $v_{S}$ is the synchrotron peak frequency), ISP intermediate synchrotron peaked $\left(10^{14} \mathrm{~Hz}<v_{\mathrm{S}}<10^{15} \mathrm{~Hz}\right)$, and HSP high synchrotron peaked $\left(v_{\mathrm{S}}>10^{15} \mathrm{~Hz}\right)$ blazars; from Abdo et al. (2010); ${ }^{(4)} \mathrm{TeV}$ Sources from TeVCat ${ }^{2}$.

References. (a) Zhang et al. (2013); Impiombato et al. (2011); D'Ammando et al. (2010a, 2011a, 2013); Pucella et al. (2010); Impiombato et al. (2008); Pian et al. (2007); Dolcini et al. (2005). (b) D'Ammando et al. (in prep.). (c) D'Ammando et al. (2011a,b,c, 2010a,b,c). (d) Covino et al. (2010); Impiombato et al. (2008); Foschini et al. (2008); Dolcini et al. (2007). (e) D’Ammando et al. (2011a,b, 2010a).

ratio in a fast-moving alt-azimuth mount that provides two stable Nasmyth focal stations. The two cameras, REMIR (Conconi et al. 2004) for near-IR and ROSS (Tosti et al. 2004) for the optical, both have a field of view of $10 \times 10$ arcmin and imaging capabilities with the usual NIR $(z, J, H$, and $K)$ and JohnsonCousins VRI filters. They allow us to obtain nearly simultaneous data. The REM software system (Covino et al. 2004) is able to manage complex observational strategies in a fully autonomous way.

In this paper we consider optical and NIR data in VRIJHK bands (from 0.55 to $2.15 \mu \mathrm{m}$ ), collected from April 11, 2005 (53 471 MJD) to June 30, 2012 (56 108 MJD). Instrumental magnitudes were obtained via aperture photometry, using aperture radii of 5 arcsec and typically with $300 \mathrm{~s}$ integration time in the optical and $150 \mathrm{~s}$ integration time in the NIR. Calibration was performed by means of comparison stars in the field reported in Two Micron All Sky Survey Catalog (2MASS) ${ }^{3}$ (Skrutskie et al. 2006) for NIR frames. For optical standard stars, calibrated sequences from several authors were followed, depending on the blazar field. Among the calibrated stars we chose a reference star, present in each frame, and a check star for each source of the sample. The check star can change depending on its positions in the frames. When the check star differed from its mean value by more than $2.5 \sigma$, the image was discarded. Dubious and mainly low-state images were visually inspected in order to remove frames affected by inhomogenous background, bad tracking, etc., to obtain a clean photometric sample. Interesting episodes were similarly carefully inspected.

\section{The blazar sample}

The seven blazars presented here (Table 1) are all bright, well studied objects. Among the sources of the class monitored by $\mathrm{REM}^{4}$ ( 60), they were chosen with the basic criterion of having the largest extensions of the total coverage. Six sources are BL Lac objects, and one is a flat spectrum radio quasar (FSRQ). The redshift is known for all of them. They are all Fermi gamma

\footnotetext{
1 http://simbad.u-strasbg.fr/

2 http://tevcat.uchicago.edu/

3 http://www.ipac. caltech.edu/2mass/

4 REM data are available at http://www . rem. inaf . it
}

ray sources and four of them were detected in the $\mathrm{TeV}$ band as shown in Table 1. Recently a strong but complex correlation between optical and gamma rays has been demonstrated (e.g. Chatterjee et al. 2013). Some REM photometry on the target sources has already been published.

In Fig. 1 we report some images of the CCD fields where the target, the reference, and the check stars are indicated. The magnitudes of the reference and the check stars are given in Table 2 for $V, R$, and $I$ optical filters. The procedure described in the previous section was followed.

\section{Long term variability}

The light curves resulting from the averaging of the single measurements on each night are presented in Fig. 2 and in Table 3. A full version of Table 3 is available at the CDS. If more data are present in a night interval, weighted averaged magnitudes are taken. The standard errors of source and of the check star added in quadrature with the mean instrumental and calibration error is assumed as uncertainty. For each source the corresponding check star light curve is reported in the $J$ filter and in the other bands the curve for the check star is very similar.

The properties of the light curves are reported in Table 4, where the flux values are given after dereddening with the coefficient reported in Table 5. All seven sources are strongly variable. The amplitude of variability is larger than four magnitudes in the NIR bands of PKS 0537-441and PKS 1510-089, decreasing in the optical. For the BL Lacs PKS 0735+17, OJ 287, PKS 2005489 , and $\mathrm{W}$ Comae, the variability is $1-2 \mathrm{mag}$, while the case of PKS 2155-304 is intermediate. The curves are spiky, with monotonic trends that can last for months. There are flares of various intensities and shapes, and their classification appears arduous. As an example we select in Fig. 3 the prominent flare that occurred in PKS 1510-089 and lasted for nine days around May 10, 2009 (54961 MJD) while the source was brightening for 120 days. The source $K$ flux dramatically rose from $\sim 10$ to $73.8 \mathrm{mJy}$ in about six days before decaying in four days to the original value. The noticeable event was also observed simultaneously by Sasada et al. (2011) and Bonning et al. (2012). 

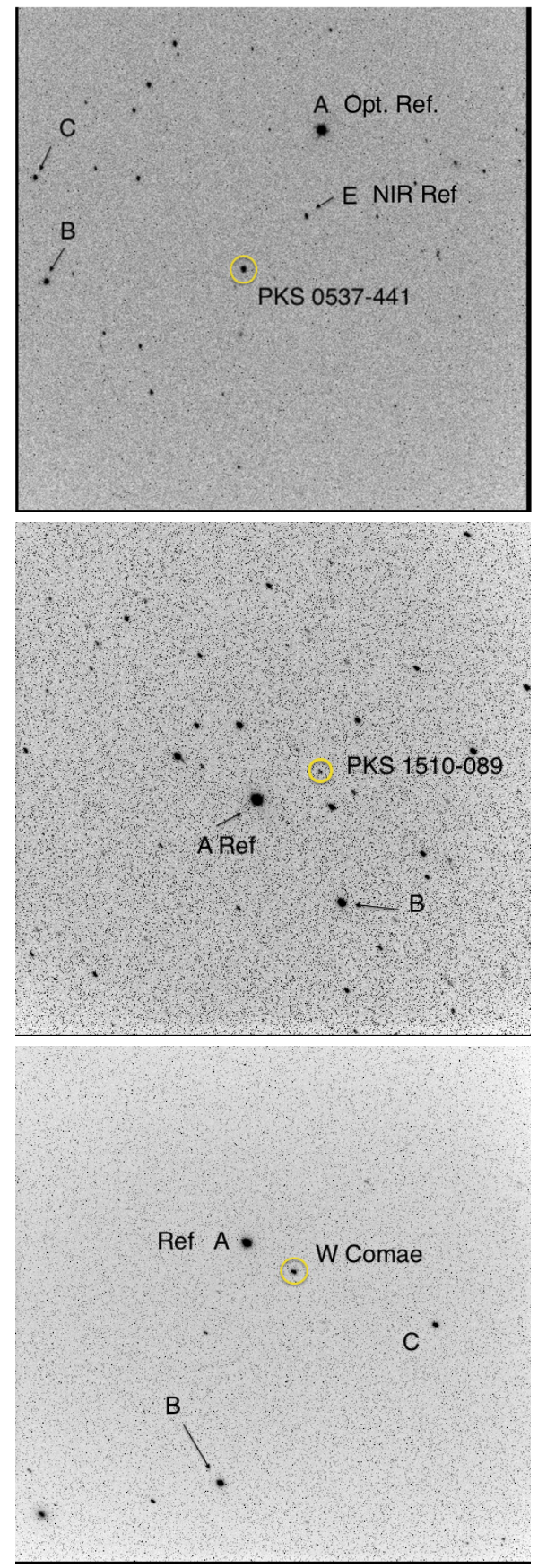

Fig. 1. Charts for the seven targets in $R$ filter.
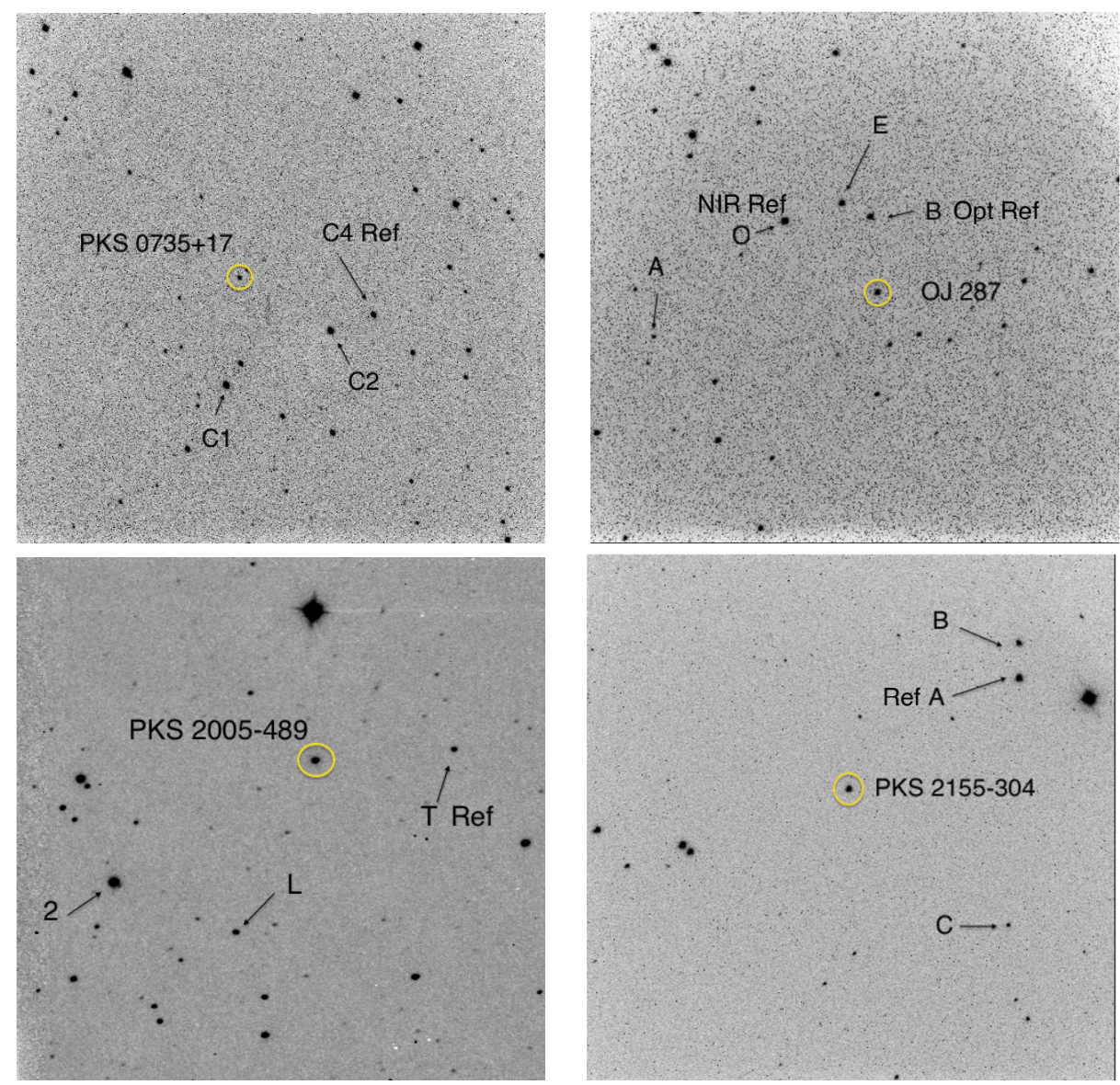

We also characterized the variability of all the light curves through the fractional variability amplitude $\sigma_{\text {rms }}$ defined as

$\sigma_{\mathrm{rms}}^{2}=\frac{1}{N \mu^{2}} \sum\left[\left(F_{i}-\mu\right)^{2}-\sigma_{i}^{2}\right]$,

where $N$ is the number of flux values $F_{i}$, with measurement uncertainties $\sigma_{i}$, and $\mu$ is the average flux. The evaluation of $\sigma_{\text {rms }}$ gives a measure of the intrinsic variability amplitude and it represents the averaged amplitude of observed variations as a percentage of the flux corrected for the effects of measurement noise. It is discussed, for example, in Nandra et al. (1997), Edelson et al. (2002), and Vaughan et al. (2003). From Table 6 and Fig. 4 it is apparent that in terms of $\sigma_{\text {rms }}$ the most variable source is PKS 1510-089, followed by PKS 0537-441 and PKS 2155-304, consistently with the variability indicated by the secular excursion of the source magnitude (see above). The dependence of $\sigma_{\text {rms }}$ on the spectral band is modest in the BL Lacs objects and is possibly in part due to the dishomogeneity in the coverage. The FSRQ PKS 1510-048 is more variable at longer wavelengths. The latter behavior with smoother trends is also pointed out by Bonning et al. (2012).

The dependence of $\sigma_{\text {rms }}$ on flux was also investigated. The $H$ and $R$ light curves were divided into four flux intervals containing approximatively the same number of points. Average fluxes and $\sigma_{\text {rms }}$ are evaluated for each interval, and no clear trend $\sigma_{\text {rms }}$ vs. $F$ is apparent. The results are collected in Table 7. We note that our results do not agree with the finding by 
PKS 0537-441

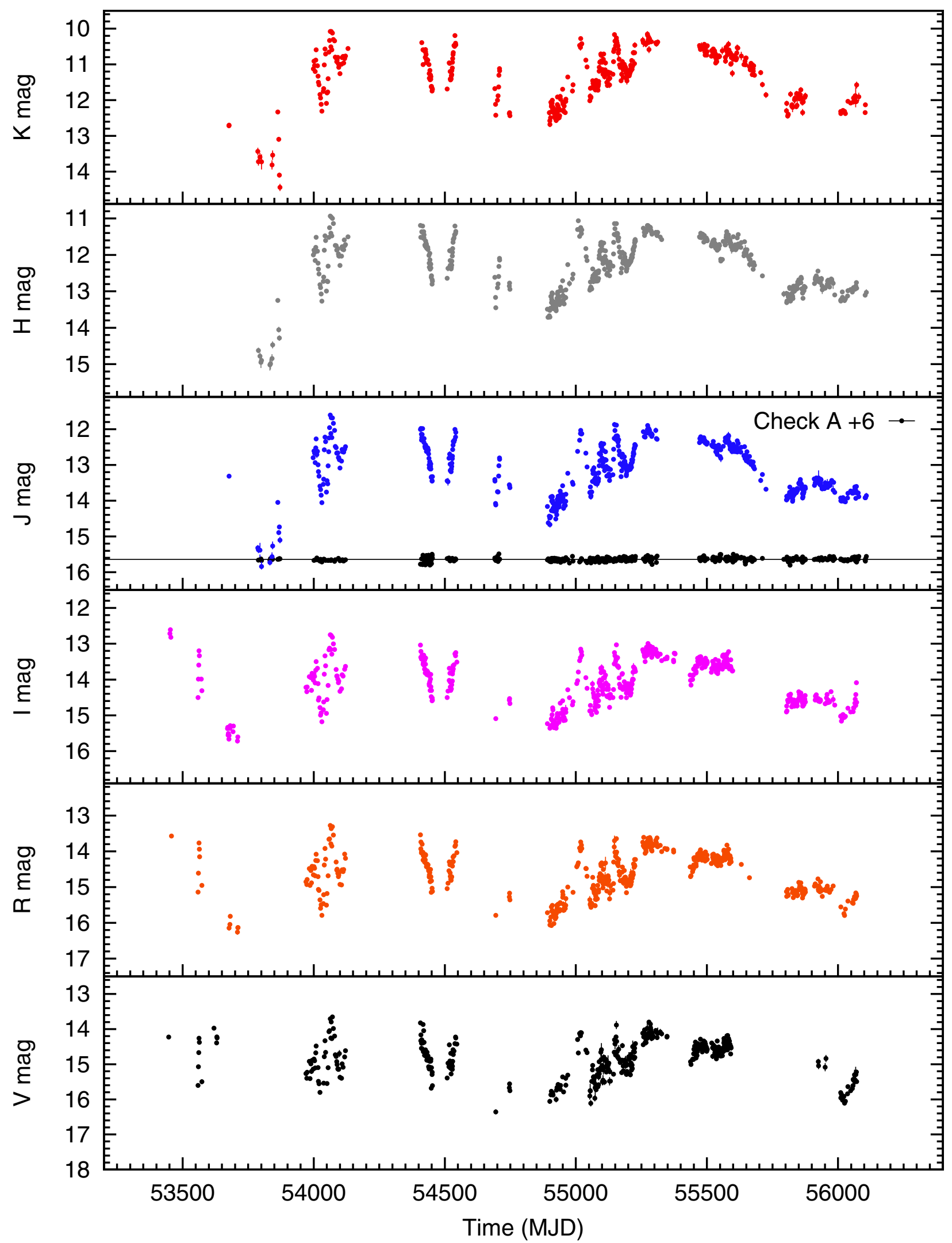

Fig. 2. REM near-infrared and optical nightly averaged light curves of the seven target sources. The light curve of the check star is reported in $J$ band (black points) with the indicated displacements $\Delta m$. 
A. Sandrinelli et al.: Variability of seven blazars in six bands

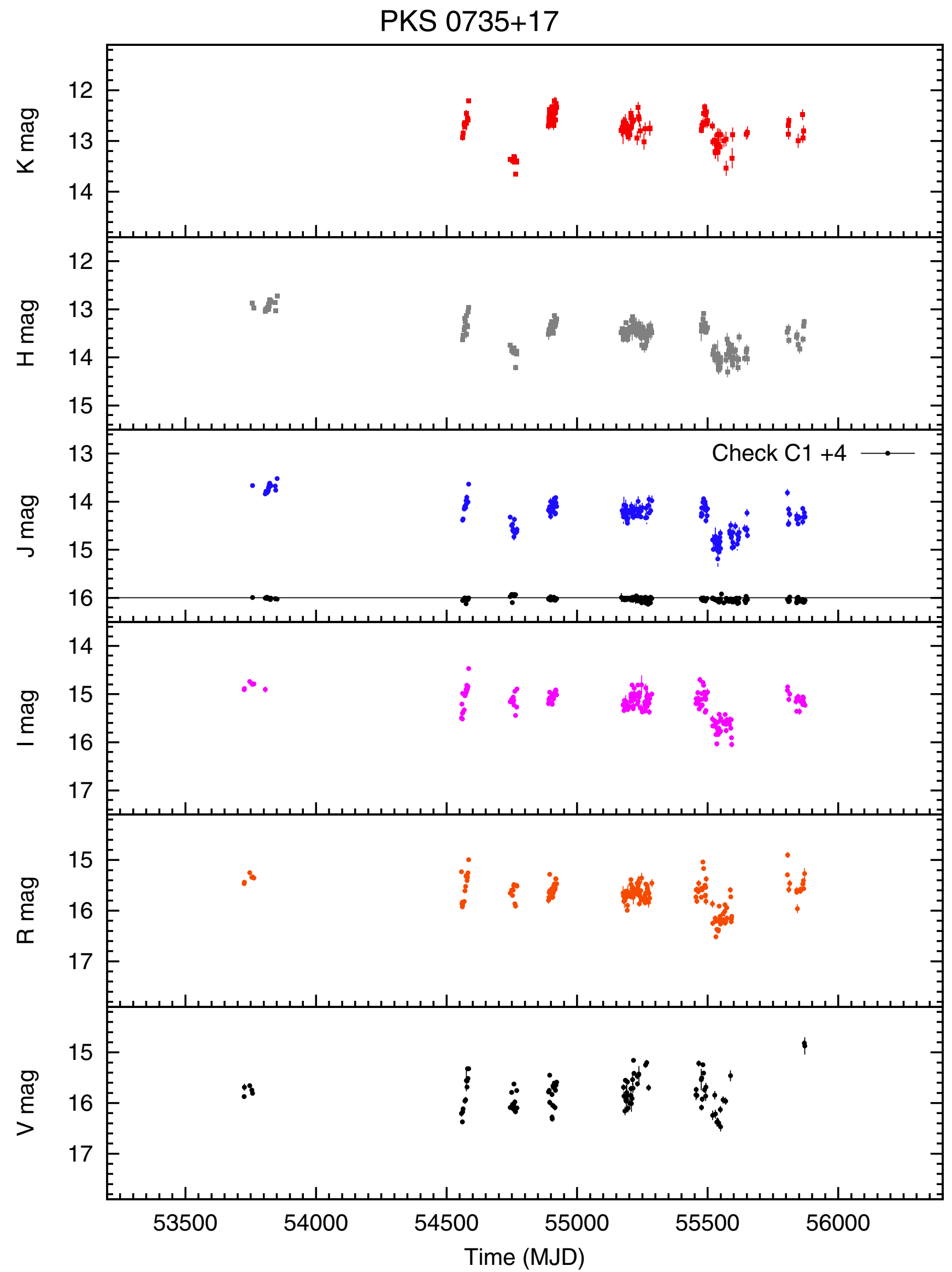

Fig. 2. continued. 


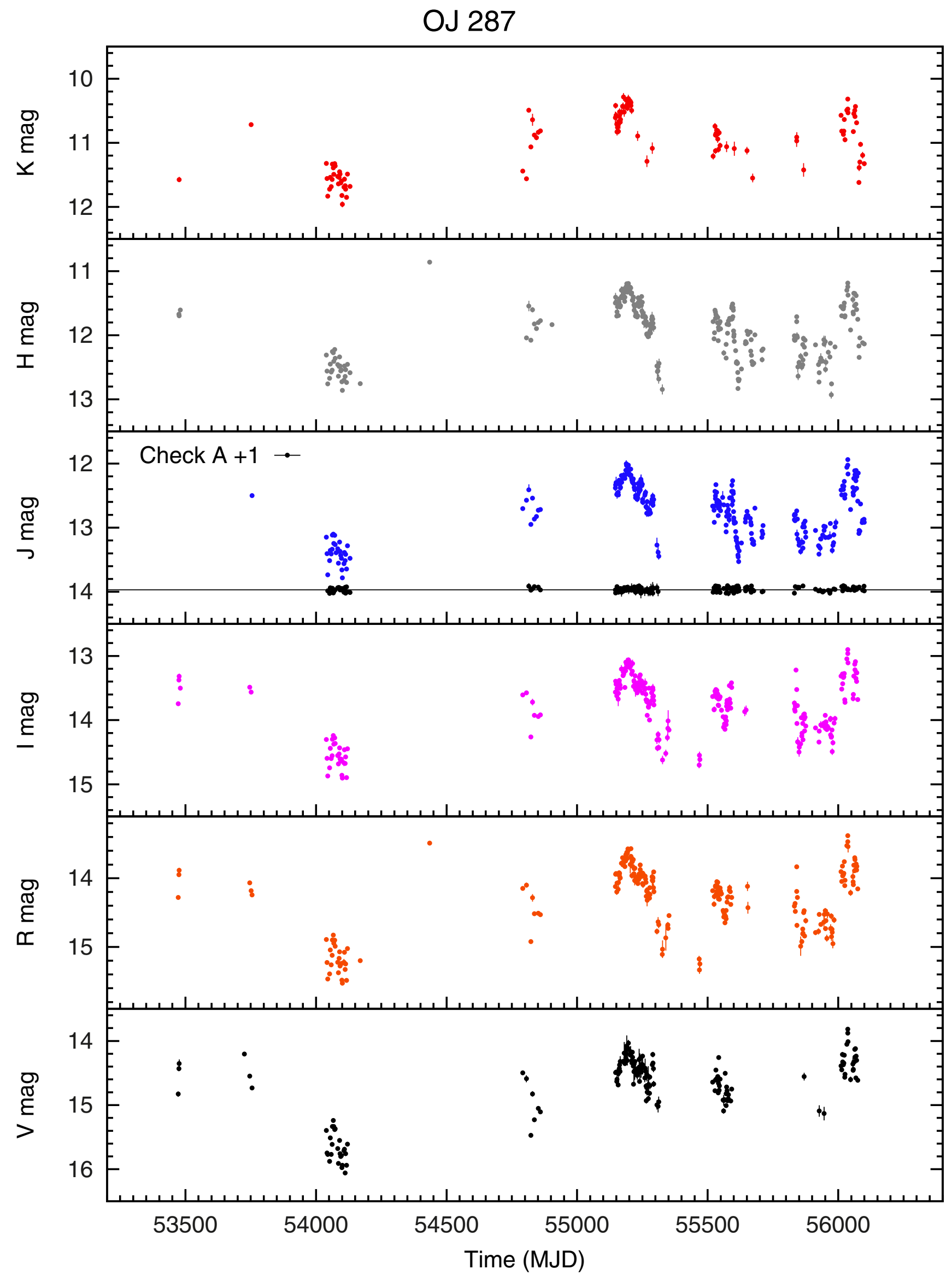

Fig. 2. continued. 
A. Sandrinelli et al.: Variability of seven blazars in six bands

PKS 1510-089

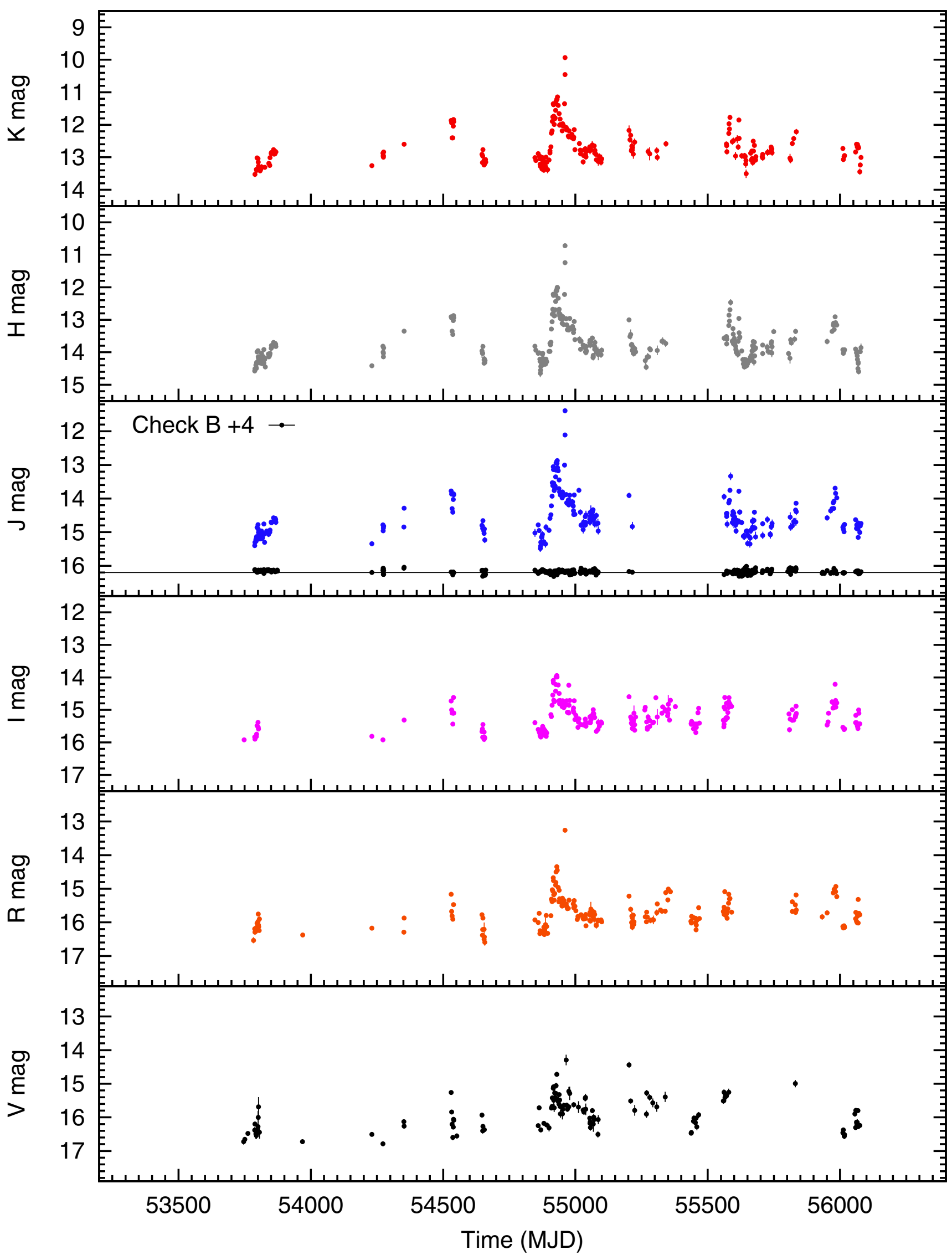

Fig. 2. continued. 
PKS 2005-489

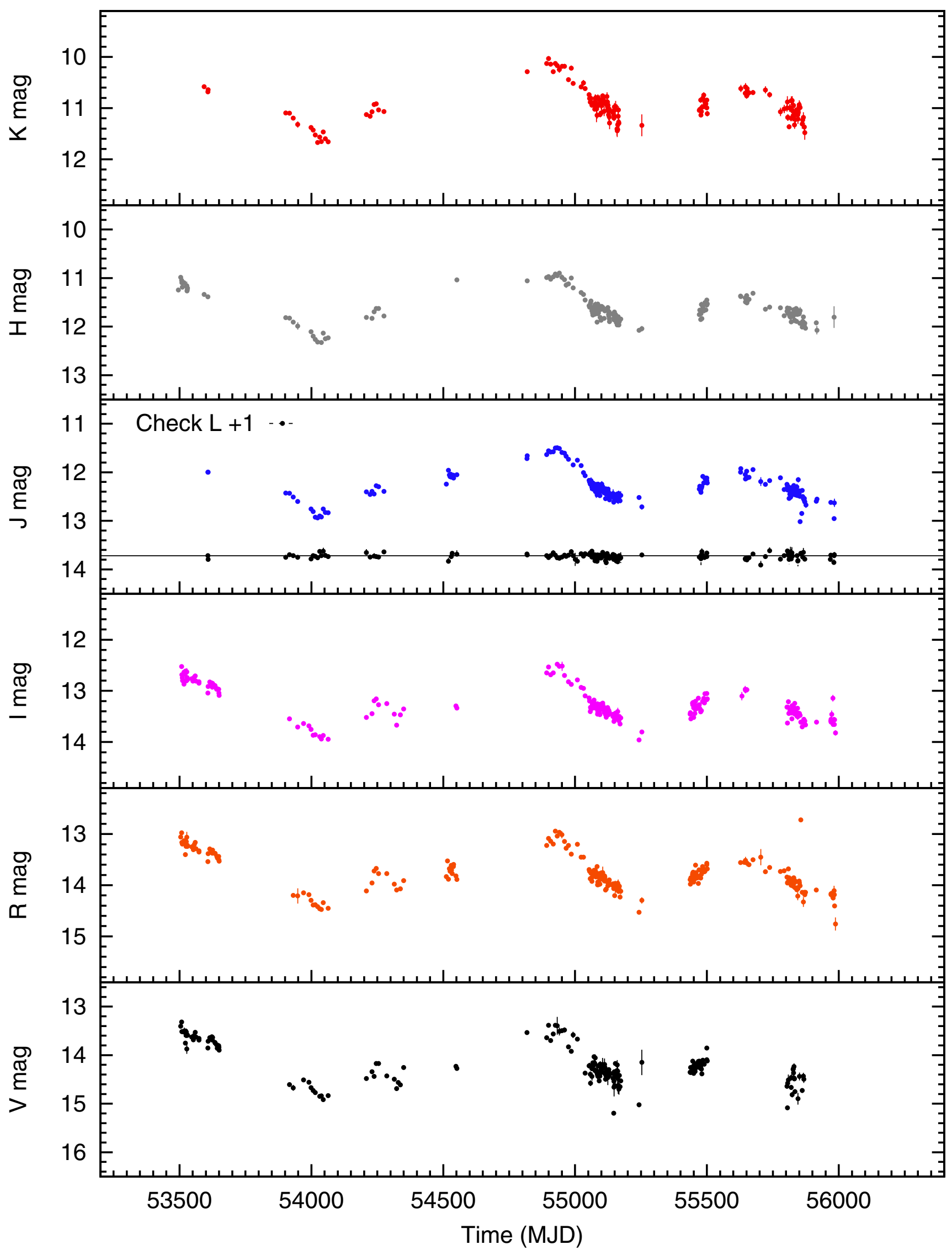

Fig. 2. continued. 
A. Sandrinelli et al.: Variability of seven blazars in six bands

PKS 2155-304

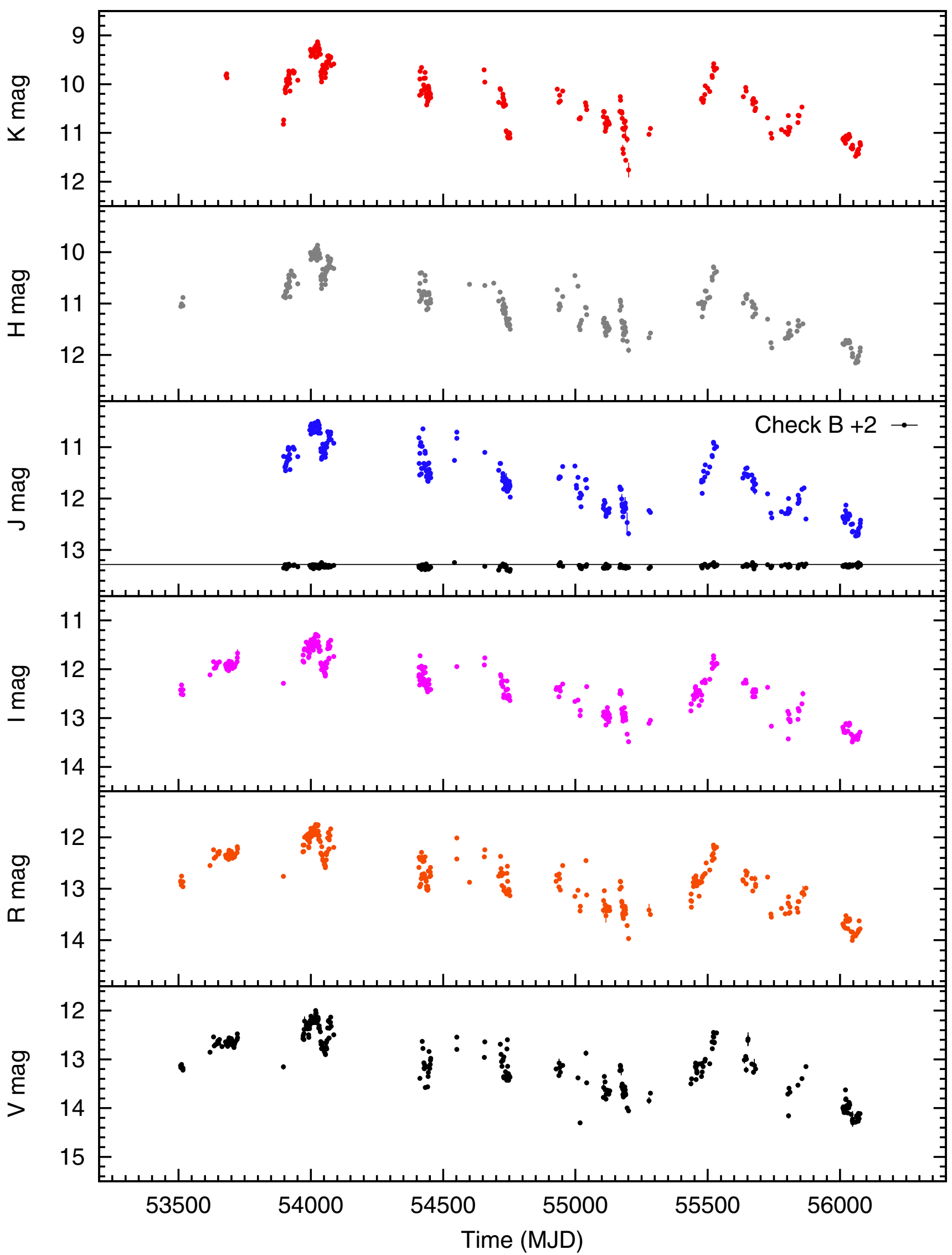

Fig. 2. continued. 


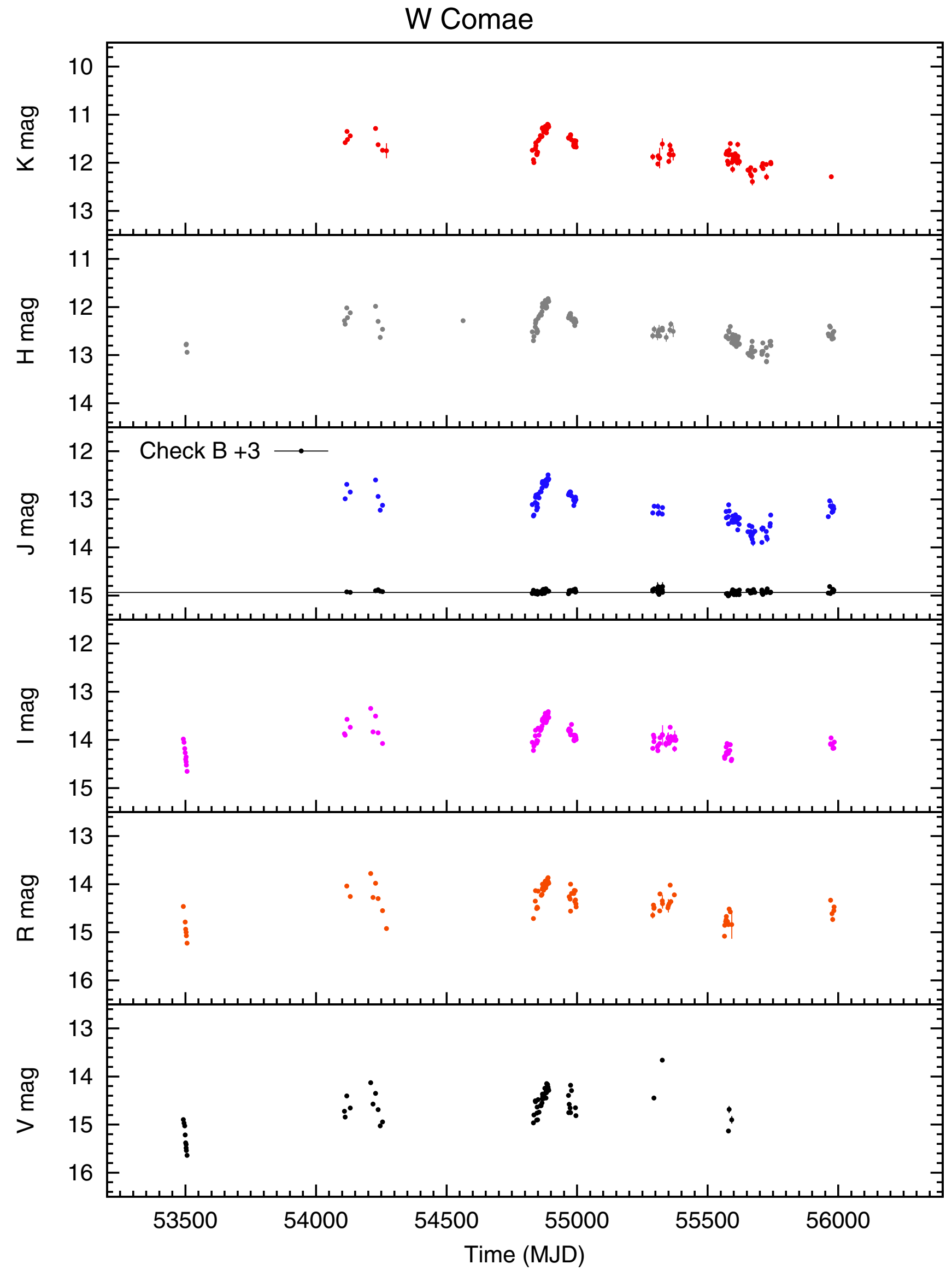

Fig. 2. continued. 
Table 2. $V, R, I$ comparison stars in the fields of our sample.

\begin{tabular}{|c|c|c|c|c|c|c|c|c|c|}
\hline Source & Star & $\begin{array}{c}\text { Coordinates } \\
\text { RA Dec } \\
\text { [h:m:s] [d:m:s] }\end{array}$ & $V$ & $\sigma(V)$ & $R$ & $\sigma(R)$ & $I$ & $\sigma(I)$ & References \\
\hline \multirow[t]{4}{*}{ PKS 0537-441 } & A (Opt Ref) & 05:39:04.51 - 44:06:38.2 & 10.680 & 0.010 & 10.400 & 0.010 & 10.120 & 0.010 & 1 \\
\hline & $\mathrm{B}$ & 05:38:49.33 - 44:01:30.0 & 13.200 & 0.010 & 12.870 & 0.010 & 12.540 & 0.010 & 1 \\
\hline & $\mathrm{C}$ & 05:38:59.97 - 44:01:19.1 & 14.080 & 0.010 & 13.710 & 0.010 & 13.340 & 0.010 & 1 \\
\hline & E (NIR Ref) & 05:38:55.70 - 44:06:19.6 & 14.843 & 0.035 & 14.151 & 0.034 & 13.585 & 0.023 & 6 \\
\hline \multirow[t]{2}{*}{ PKS 0735+17 } & $\mathrm{C} 1$ & $07: 38: 00.57+17: 41: 19.7$ & 13.26 & 0.04 & 12.89 & 0.04 & 12.57 & 0.04 & 2 \\
\hline & C2 (Ref) & 07:38:08.55 + 17:40:29.2 & 13.31 & 0.04 & 12.79 & 0.04 & 12.32 & 0.04 & 2 \\
\hline \multirow[t]{3}{*}{ OJ 287} & O (NIR Ref) & $08: 54: 53.38+20: 04: 44.8$ & 14.192 & 0.003 & 13.707 & 0.002 & 13.262 & 0.004 & 3 \\
\hline & B (Opt Ref) & $08: 54: 54.45+20: 06: 13.6$ & 14.627 & 0.003 & 14.315 & 0.003 & 13.999 & 0.004 & 3 \\
\hline & $\mathrm{E}$ & $08: 54: 55.20+20: 05: 42.3$ & 14.974 & 0.003 & 14.632 & 0.003 & 14.304 & 0.004 & 3 \\
\hline \multirow[t]{2}{*}{ PKS 1510-089 } & A (Ref) & $15: 12: 46.16-09: 05: 23.0$ & 11.571 & 0.001 & 11.195 & 0.002 & 10.847 & 0.001 & 3 \\
\hline & $\mathrm{B}$ & $15: 12: 53.19-09: 03: 42.4$ & 13.282 & 0.016 & 12.992 & 0.022 & 12.687 & 0.019 & 3 \\
\hline \multirow[t]{3}{*}{ PKS 2005-489 } & $\mathrm{T}$ (Ref) & $20: 09: 40: 03-48: 50: 21.8$ & 14.92 & - & 14.39 & - & 13.86 & 0.06 & $4(V, R$ band $) ; 6$ ( $I$ band $)$ \\
\hline & $\mathrm{L}$ & 20:09:19.04 - 48:46:43.1 & 14.41 & - & 13.89 & - & 13.45 & 0.09 & $4(V, R$ band $) ; 6$ ( $I$ band $)$ \\
\hline & 2 & 20:09:05.42 - 48:47:20.8 & 11.92 & 0.03 & 11.33 & 0.03 & 10.79 & 0.03 & 6 \\
\hline \multirow[t]{3}{*}{ PKS 2155-304 } & A (Ref) & 21:59:02.49 - 30:10:46.6 & 12.050 & 0.010 & 11.670 & 0.010 & 11.300 & 0.020 & 1 \\
\hline & $\mathrm{B}$ & 21:59:05.34 - 30:10:51.0 & 13.000 & 0.010 & 12.470 & 0.020 & 12.020 & 0.020 & 1 \\
\hline & $\mathrm{C}$ & $21: 58: 42.33-30: 10: 27.4$ & 14.280 & 0.010 & 13.920 & 0.020 & 13.560 & 0.020 & 1 \\
\hline \multirow[t]{3}{*}{ W Comae } & A (Ref) & $12: 21: 33.66+28: 13: 04.1$ & 12.08 & 0.02 & 11.72 & 0.04 & 11.40 & 0.04 & 5 \\
\hline & $\mathrm{B}$ & $12: 21: 13.83+28: 13: 04.3$ & 13.03 & 0.07 & 12.69 & 0.05 & 12.37 & 0.05 & 6 \\
\hline & $\mathrm{C}$ & $12: 21: 28.61+28: 16: 37.7$ & 14.81 & 0.04 & 13.86 & 0.04 & 12.68 & 0.04 & 5 \\
\hline
\end{tabular}

Notes. Stars used as references are marked with Ref; NIR Ref and Opt Ref specify that the reference stars change between NIR and optical bands. The check star can change depending on its positions in the frames.

References. (1) Hamuy \& Maza (1989); (2) Ciprini et al. (2007); (3) González-Pérez et al. (2001); (4) Rector \& Perlman (2003); (5) Fiorucci \& Tosti (1996); (6) this work.

Table 3. Photometry of the seven target objects.

\begin{tabular}{lcccc}
\hline \hline Source & Filter & $\begin{array}{c}\text { Time of } \\
\text { observation } \\
{[\mathrm{MJD}]}\end{array}$ & $\begin{array}{c}\text { Average } \\
\text { magnitude } \\
{[\mathrm{mag}]}\end{array}$ & $\begin{array}{c}\text { Magnitude } \\
\text { error } \\
{[\mathrm{mag}]}\end{array}$ \\
\hline PKS0537-441 & $K$ & 53453 & 10.685 & 0.031 \\
PKS0537-441 & $K$ & 53676 & 12.716 & 0.044 \\
PKS0537-441 & $K$ & 53677 & 12.699 & 0.049 \\
$\ldots .$. & $\ldots$. & $\ldots$. & $\ldots$. & $\ldots$. \\
\hline
\end{tabular}

Notes. The full table is available at the CDS.

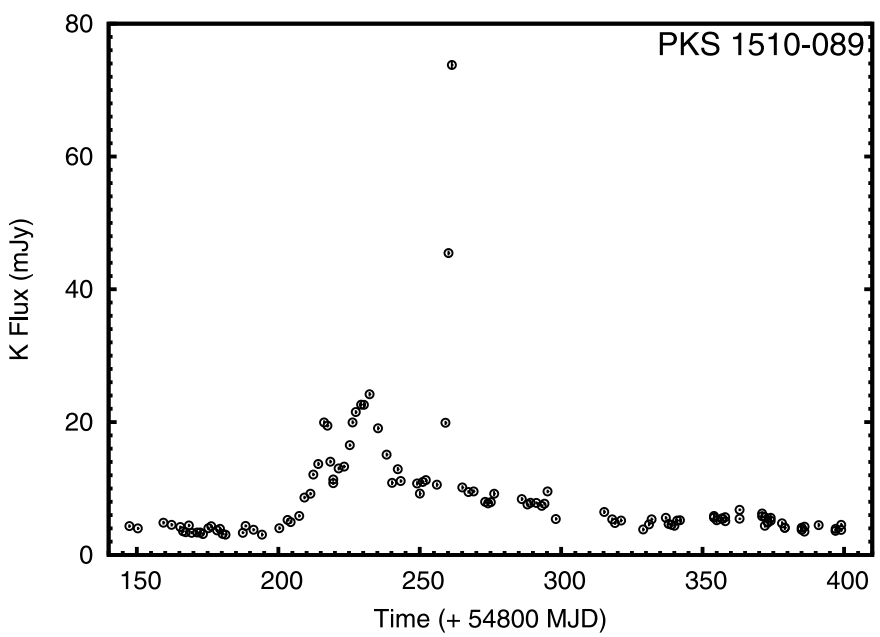

Fig. 3. Active state of PKS 1510-089 and the flare occurring around 54961 MJD.
Edelson et al. (2013) of a clear correlation between rms and flux of the BL Lac object W2R1926+42 observed by Kepler satellite.

For each source the general behavior of the light curves is similar in the various filters. Nevertheless, color-intensity plots are of some interest. We selected epochs when the time lapse between $R$ and $H$ observations was less than six minutes. The $R-H$ color vs. the $H$ magnitude is reported in Figs. 5 and 6. It is apparent that the plots are rather different. PKS 1510-089 exhibits a banana-like shape, with a general trend indicating bluer color for decreasing flux. The same tendency appears in PKS 0537441, and in PKS 0735+17. On the other hand, OJ 287 and PKS 2005-489 trace a circular spot, or rather an atoll shape. In the case of PKS 2155-304 the spot is distorted in the intensity direction. The shape of the color-intensity plots may be very different if one only considers a fraction of the overall collection of data (see the bars at the right of the figures).

The case of the flare of PKS 1510-089 around 54961 MJD marked in Fig. 5 is illustrative of the complexity of the 
Table 4. Properties of the light curves.

\begin{tabular}{|c|c|c|c|c|c|c|c|c|c|c|}
\hline Source & $\begin{array}{c}\text { Timeframe } \\
\text { in } H \text { band } \\
\text { ([MJD]) }\end{array}$ & Filter & $\begin{array}{c}\text { Magnitude } \\
\text { range } \\
{[\mathrm{mag}]}\end{array}$ & $\begin{array}{l}\text { Variability } \\
\text { amplitude } \\
\Delta m[\mathrm{mag}]\end{array}$ & $\begin{array}{c}\text { Average } \\
\text { magnitude } \\
{[\mathrm{mag}]}\end{array}$ & $\begin{array}{l}\text { Mode } 1 \\
{[\mathrm{mag}]}\end{array}$ & {$[\mathrm{mag}]$} & $\begin{array}{c}\text { Standard } \\
\text { deviation } \\
{[\mathrm{mag}]}\end{array}$ & $\begin{array}{c}\text { Flux range } \\
\text { [mJy] }\end{array}$ & $F_{\max } / F_{\min }$ \\
\hline \multirow[t]{6}{*}{ PKS 0537-44 } & $2005 / 11 / 03-2012 / 06 / 30$ & $K$ & $10.06-14.57$ & 4.51 & 11.30 & 10.6 & 11.15 & 0.74 & $1.14-63.07$ & 55.32 \\
\hline & $(53677-56108)$ & $H$ & $10.93-15.45$ & 4.52 & 12.28 & 11.8 & 12.18 & 0.73 & $0.70-45.16$ & 64.51 \\
\hline & & $J$ & $11.58-15.84$ & 4.26 & 13.09 & 12.5 & 13.01 & 0.71 & $0.74-37.41$ & 50.55 \\
\hline & & $I$ & $12.59-15.75$ & 3.16 & 14.08 & 13.6 & 14.06 & 0.63 & $1.29-23.79$ & 18.44 \\
\hline & & $R$ & $13.27-16.28$ & 3.01 & 14.65 & 14.2 & 14.65 & 0.61 & $1.04-16.64$ & 16.00 \\
\hline & & $V$ & $13.11-16.36$ & 3.25 & 14.83 & 14.6 & 14.78 & 0.53 & $1.18-23.49$ & 19.91 \\
\hline \multirow[t]{6}{*}{ PKS $0735+17$} & 2006/01/19-2011/11/05 & $K$ & $12.14-13.65$ & 1.51 & 12.78 & 12.7 & 12.71 & 0.32 & $2.35-9.41$ & 4.00 \\
\hline & $(53754-55870)$ & $H$ & $12.72-14.30$ & 1.58 & 13.52 & 13.4 & 13.46 & 0.34 & $2.02-8.65$ & 4.28 \\
\hline & & $J$ & $13.52-15.19$ & 1.67 & 14.28 & 14.2 & 14.23 & 0.34 & $1.34-6.27$ & 4.68 \\
\hline & & $I$ & $14.47-16.04$ & 1.57 & 15.19 & 15.1 & 15.14 & 0.28 & $0.98-4.20$ & 4.29 \\
\hline & & $R$ & $14.90-16.52$ & 1.62 & 15.68 & 15.6 & 15.64 & 0.28 & $0.83-3.68$ & 4.43 \\
\hline & & $V$ & $14.72-16.46$ & 1.74 & 15.79 & 15.7 & 15.79 & 0.32 & $1.05-5.24$ & 4.99 \\
\hline \multirow[t]{6}{*}{ OJ 287} & $2005 / 04 / 11-2012 / 06 / 21$ & $K$ & $10.27-11.96$ & 1.69 & 10.99 & 10.4 & 10.88 & 0.46 & $11.12-52.94$ & 4.76 \\
\hline & $(53471-56099)$ & $H$ & $10.84-12.93$ & 2.09 & 11.92 & 11.7 & 11.84 & 0.44 & $7.10-48.82$ & 6.88 \\
\hline & & $J$ & $11.94-13.78$ & 1.84 & 12.74 & 12.3 & 12.69 & 0.42 & $4.90-26.76$ & 5.46 \\
\hline & & $I$ & $12.90-14.90$ & 2.00 & 13.79 & 13.5 & 13.70 & 0.45 & $2.80-17.64$ & 6.30 \\
\hline & & $R$ & $13.38-15.52$ & 2.14 & 14.31 & 14.0 & 14.19 & 0.48 & $2.05-14.74$ & 7.19 \\
\hline & & $V$ & $13.82-16.06$ & 2.24 & 14.69 & 14.4 & 14.55 & 0.49 & $1.51-11.91$ & 7.89 \\
\hline \multirow{6}{*}{\multicolumn{2}{|c|}{$\begin{array}{c}\text { PKS } 1510-0892006 / 01 / 27-2012 / 06 / 01 \\
(53783-56079)\end{array}$}} & $K$ & $9.93-13.78$ & 3.85 & 12.67 & 12.8 & 12.82 & 0.56 & $2.14-73.8$ & 34.49 \\
\hline & & $H$ & $10.72-14.80$ & 4.08 & 13.74 & 14.0 & 13.89 & 0.59 & $1.31-56.58$ & 43.19 \\
\hline & & $J$ & $11.39-15.57$ & 4.18 & 14.54 & 14.7 & 14.69 & 0.61 & $1.00-47.17$ & 47.17 \\
\hline & & $I$ & $13.95-15.97$ & 2.02 & 15.21 & 15.4 & 15.28 & 0.40 & $1.18-7.62$ & 6.46 \\
\hline & & $R$ & $13.26-16.69$ & 3.43 & 15.73 & 15.9 & 15.79 & 0.44 & $0.83-19.44$ & 23.42 \\
\hline & & V & $14.29-16.93$ & 2.64 & 15.90 & 16.2 & 15.94 & 0.50 & $0.83-9.44$ & 11.37 \\
\hline \multirow{6}{*}{\multicolumn{2}{|c|}{$\begin{array}{c}\text { PKS 2005-489 2005/04/20-2012/02/25 } \\
(53480-55982)\end{array}$}} & $K$ & $10.03-11.67$ & 1.64 & 10.96 & 11.1 & 10.98 & 0.32 & $14.61-66.42$ & 4.55 \\
\hline & & $H$ & $10.88-12.33$ & 1.45 & 11.64 & 11.7 & 11.69 & 0.29 & $12.55-47.53$ & 3.79 \\
\hline & & $J$ & $11.45-13.01$ & 1.56 & 12.32 & 12.4 & 12.37 & 0.28 & $10.17-42.85$ & 4.21 \\
\hline & & $I$ & $12.09-13.96$ & 1.87 & 13.27 & 13.3 & 13.34 & 0.31 & $6.99-38.97$ & 5.58 \\
\hline & & $R$ & $12.72-14.76$ & 2.04 & 13.76 & 13.9 & 13.83 & 0.34 & $4.42-28.81$ & 6.52 \\
\hline & & $V$ & $12.98-15.20$ & 2.22 & 14.19 & 14.2 & 14.27 & 0.39 & $3.61-27.92$ & 7.73 \\
\hline \multirow{6}{*}{ PKS 2155-304 } & 2005/05/18-2012/05/29 & $K$ & $9.08-11.57$ & 2.49 & 10.03 & 9.3 & 10.55 & 0.62 & $15.92-157.49$ & 9.89 \\
\hline & $(53508-56076)$ & $H$ & $9.81-12.16$ & 2.35 & 10.94 & 11.6 & 11.43 & 0.62 & $14.40-124.96$ & 8.68 \\
\hline & & $J$ & $10.44-12.75$ & 2.32 & 11.40 & 10.6 & 11.35 & 0.63 & $12.57-106.07$ & 8.44 \\
\hline & & $I$ & $11.17-13.53$ & 2.36 & 11.92 & 11.9 & 11.90 & 0.37 & $9.78-85.69$ & 8.77 \\
\hline & & $R$ & $11.63-14.04$ & 2.41 & 12.40 & 12.3 & 12.32 & 0.41 & $7.88-72.53$ & 9.20 \\
\hline & & $V$ & $11.98-14.30$ & 2.32 & 12.68 & 12.6 & 12.63 & 0.39 & $7.49-64.43$ & 8.47 \\
\hline \multirow[t]{6}{*}{ W Comae } & $2005 / 05 / 12-2012 / 02 / 26$ & $K$ & $11.20-12.39$ & 1.19 & 11.71 & 11.3 & 11.72 & 0.30 & $7.47-22.42$ & 3.00 \\
\hline & $(53502-55983)$ & $H$ & $11.83-13.14$ & 1.31 & 12.48 & 12.7 & 12.53 & 0.33 & $5.84-19.60$ & 3.36 \\
\hline & & $J$ & $12.49-14.15$ & 1.66 & 13.17 & 13.4 & 13.17 & 0.37 & $3.48-16.07$ & 4.62 \\
\hline & & $I$ & $13.35-14.67$ & 1.33 & 13.93 & 14.0 & 13.97 & 0.28 & $3.42-11.61$ & 3.39 \\
\hline & & $R$ & $13.78-15.24$ & 1.46 & 14.36 & 14.0 & 14.34 & 0.33 & $2.64-10.11$ & 3.83 \\
\hline & & V & $13.97-15.66$ & 1.69 & 14.65 & 14.4 & 14.64 & 0.39 & $2.16-10.22$ & 4.73 \\
\hline
\end{tabular}

Table 5. Absorption coefficients.

\begin{tabular}{ccccccccc}
\hline \hline & $\lambda[\mu \mathrm{m}]$ & PKS 0537-441 & PKS 0735+17 & OJ 287 & PKS 1510-089 & PKS 2005-489 & PKS 2155-304 & W Comae \\
\hline $\mathrm{A}_{\mathrm{K}}$ & 2.15 & 0.0137 & 0.0124 & 0.0103 & 0.0369 & 0.0205 & 0.0080 & 0.0085 \\
$\mathrm{~A}_{H}$ & 1.64 & 0.0212 & 0.0192 & 0.0160 & 0.0571 & 0.0317 & 0.0124 & 0.0132 \\
$\mathrm{~A}_{J}$ & 1.25 & 0.0328 & 0.0297 & 0.0248 & 0.0883 & 0.0491 & 0.0192 & 0.0204 \\
$\mathrm{~A}_{I}$ & 0.804 & 0.0685 & 0.0620 & 0.0517 & 0.1845 & 0.1026 & 0.0400 & 0.0426 \\
$\mathrm{~A}_{R}$ & 0.659 & 0.0946 & 0.0856 & 0.0714 & 0.2548 & 0.1417 & 0.0552 & 0.0589 \\
$\mathrm{~A}_{V}$ & 0.55 & 0.1162 & 0.1051 & 0.0878 & 0.3128 & 0.1740 & 0.0678 & 0.0723 \\
\hline
\end{tabular}

Notes. Extinction values are derived from the NASA/IRPAC Infrared Science Archive (Schlegel et al. 1998). Cardelli et al. (1989) formulae were used to calculate the absorption coefficients, assuming the extinction to reddening ratio $A v / E(B-V)=3.1$. Those formulae are available at http://dogwood.physics.mcmaster.ca/Acurve.html 
A. Sandrinelli et al.: Variability of seven blazars in six bands

Table 6. NIR-optical fractional variability amplitude $\sigma_{\text {rms }}$ of the blazar sample $(\times 100 \%)$.

\begin{tabular}{lccrrrcc}
\hline \hline Source & Class & \multicolumn{1}{c}{$K$} & \multicolumn{1}{c}{$H$} & \multicolumn{1}{c}{$J$} & $I$ & $R$ & $V$ \\
\hline PKS 0537-441 & BL Lac & $54 \pm 2$ & $57 \pm 2$ & $57 \pm 2$ & $55 \pm 2$ & $52 \pm 2$ & $47 \pm 2$ \\
PKS 0735+17 & BL Lac & $25 \pm 2$ & $30 \pm 2$ & $29 \pm 2$ & $23 \pm 1$ & $25 \pm 1$ & $31 \pm 2$ \\
OJ 287 & BL Lac & $39 \pm 3$ & $38 \pm 2$ & $37 \pm 2$ & $37 \pm 2$ & $39 \pm 2$ & $36 \pm 2$ \\
PKS 1510-089 & FSRQ & $92 \pm 4$ & $104 \pm 4$ & $115 \pm 5$ & $43 \pm 2$ & $69 \pm 4$ & $52 \pm 4$ \\
PKS 1510-089 & & $61 \pm 3$ & $69 \pm 3$ & $68 \pm 3$ & $44 \pm 2$ & $45 \pm 2$ & $47 \pm 4$ \\
PKS 2005-489 & BL Lac & $32 \pm 2$ & $29 \pm 2$ & $29 \pm 1$ & $31 \pm 1$ & $33 \pm 1$ & $38 \pm 2$ \\
PKS 2155-30 & BL Lac & $57 \pm 3$ & $54 \pm 2$ & $54 \pm 2$ & $48 \pm 2$ & $49 \pm 2$ & $49 \pm 2$ \\
W Comae & BL Lac & $26 \pm 2$ & $31 \pm 2$ & $33 \pm 2$ & $25 \pm 2$ & $28 \pm 2$ & $34 \pm 3$ \\
\hline
\end{tabular}

Notes. ${ }^{(a)}$ All data; ${ }^{(b)}$ because of inhomogenous sampling, the flare occurring on 54 956-54 969 MJD was removed.

Table 7. NIR-optical fractional variability amplitude $\sigma_{\text {rms }}$ dependence on flux.

\begin{tabular}{|c|c|c|c|c|c|c|c|c|}
\hline \multicolumn{3}{|c|}{ PKS 0537-441 } & \multicolumn{3}{|c|}{ PKS 1510-089 } & \multicolumn{3}{|c|}{ PKS 2155-304 } \\
\hline Filter & $\begin{array}{c}\text { Average flux } \\
{[\mathrm{mJy}]}\end{array}$ & $\begin{array}{c}\begin{array}{c}\sigma_{\text {rms }} \\
(\times 100 \%)\end{array} \\
\end{array}$ & Filter & $\begin{array}{c}\text { Average flux } \\
{[\mathrm{mJy}]}\end{array}$ & $\begin{array}{c}\begin{array}{c}\sigma_{\text {rms }} \\
(\times 100 \%)\end{array} \\
\end{array}$ & Filter & $\begin{array}{c}\text { Average flux } \\
{[\mathrm{mJy}]}\end{array}$ & $\begin{array}{c}\sigma_{\text {rms }} \\
(\times 100 \%)\end{array}$ \\
\hline \multirow[t]{4}{*}{$H$} & $5.7 \pm 0.13$ & $27.5 \pm 1.7$ & \multirow[t]{4}{*}{$H$} & $2.08 \pm 0.02$ & $7.9 \pm 1.1$ & \multirow[t]{4}{*}{$H$} & $22.6 \pm 0.5$ & $17.3 \pm 1.6$ \\
\hline & $10.6 \pm 0.2$ & $19.0 \pm 1.2$ & & $2.74 \pm 0.02$ & - & & $35.7 \pm 0.6$ & $13.7 \pm 1.2$ \\
\hline & $18.4 \pm 0.2$ & $12.3 \pm 0.8$ & & $3.53 \pm 0.04$ & $6.3 \pm 1.0$ & & $54 \pm 1$ & $15.6 \pm 1.4$ \\
\hline & $27.1 \pm 0.3$ & $15.1 \pm 0.8$ & & $8.8 \pm 0.8$ & $81 \pm 7$ & & $91 \pm 2$ & $16.5 \pm 1.5$ \\
\hline \multirow[t]{4}{*}{$R$} & $2.31 \pm 0.05$ & $23.8 \pm 1.5$ & \multirow[t]{4}{*}{$R$} & $1.31 \pm 0.02$ & $10.2 \pm 0.3$ & \multirow[t]{4}{*}{$R$} & $13.0 \pm 0.3$ & $17.0 \pm 1.4$ \\
\hline & $3.80 \pm 0.04$ & $11.5 \pm 0.9$ & & $1.73 \pm 0.01$ & - & & $21.7 \pm 0.2$ & $9.4 \pm 0.8$ \\
\hline & $6.14 \pm 0.06$ & $11.1 \pm 0.8$ & & $2.14 \pm 0.02$ & $5.9 \pm 1.0$ & & $31.8 \pm 0.5$ & $13.7 \pm 1.1$ \\
\hline & $9.3 \pm 0.2$ & $19.0 \pm 1.2$ & & $3.9 \pm 0.4$ & $64 \pm 7$ & & $48.4 \pm 0.9$ & $16.0 \pm 1.3$ \\
\hline
\end{tabular}

Notes. No evaluation is reported when $\sigma_{\text {rms }}^{2}$ is dominated by errors, resulting in a negative value.

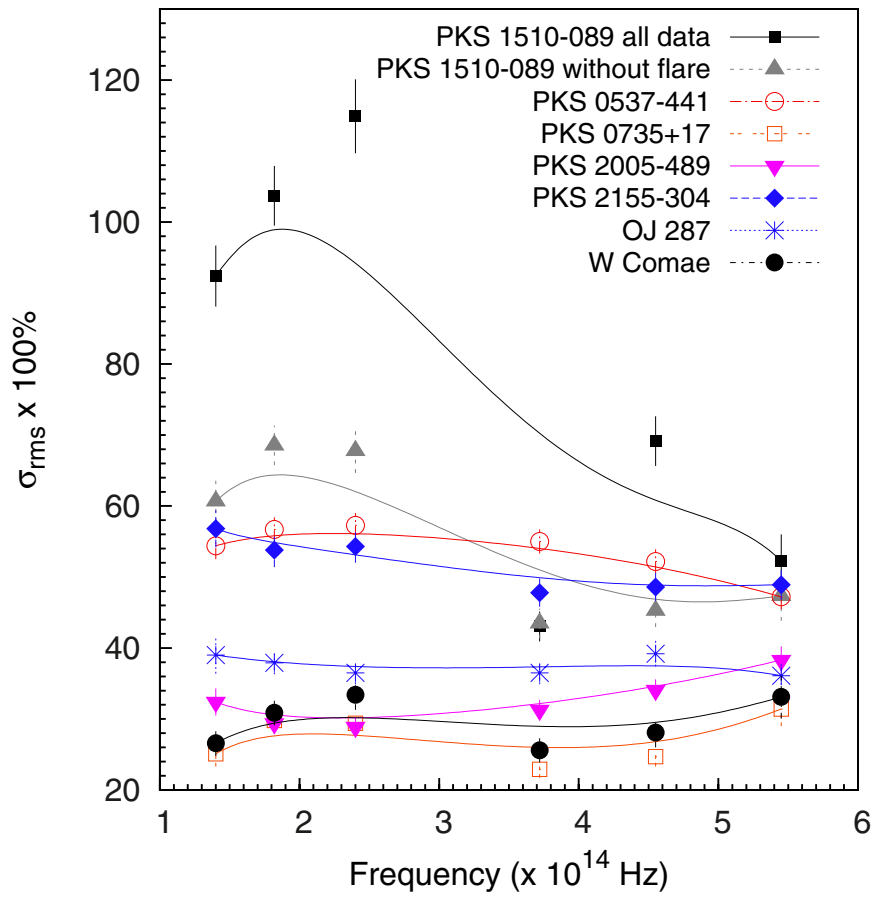

Fig. 4. Fractional variability amplitude vs. frequency. The lines are a help to the eye.

color-intensity dependence. During both the rising and falling phases of the flare, $R-H$ remained constant and $J-K$ was decreasing by $\sim 0.5$, indicating a bluer color for increasing intensity. This result may be relevant in order to reconcile the achromaticity of the flare reported by Bonning et al. (2012) on the basis of the $B-J$ color vs. $J$, and the suggestion by

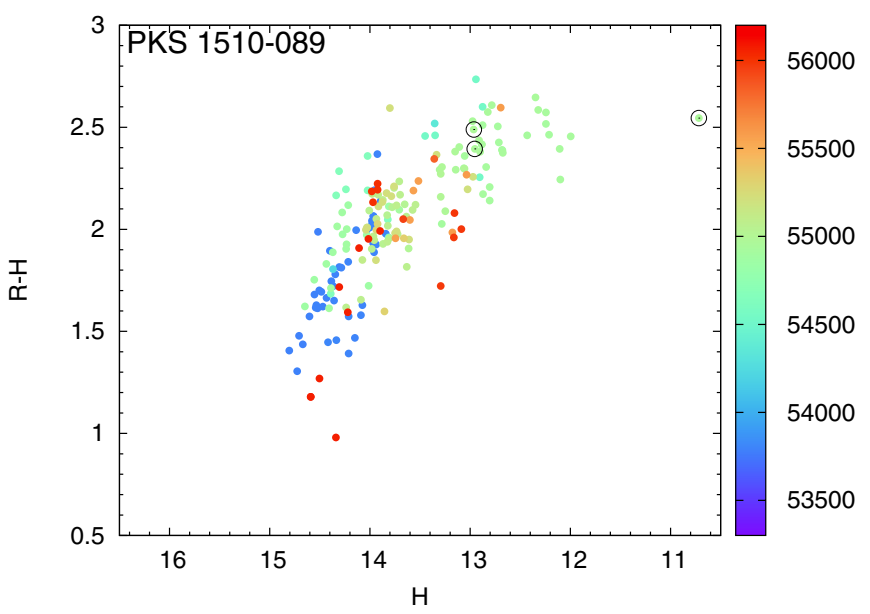

Fig. 5. Color-intensity diagram for PKS 1510-089. The color of each point is related to the epoch of detection, according to the bar on the right. The circles encompass the points referring to the flare observed around 54961 MJD. Error bars are omitted for readability.

Sasada et al. (2011) of bluer values for higher intensity indicated by the $V-J$ vs. $V$ plot.

The availability of six filters allows the construction of the spectral flux distributions and the SEDs that are reported in Fig. 7. In all sources the highest states are well fitted by a single power law, and deviations may appear in the lowest states.

\section{Rapid events in the light curves}

REM observations of blazars, and specifically of the seven sources considered here, contain data deriving from proposals with various scientific goals. A large fraction of the observations 

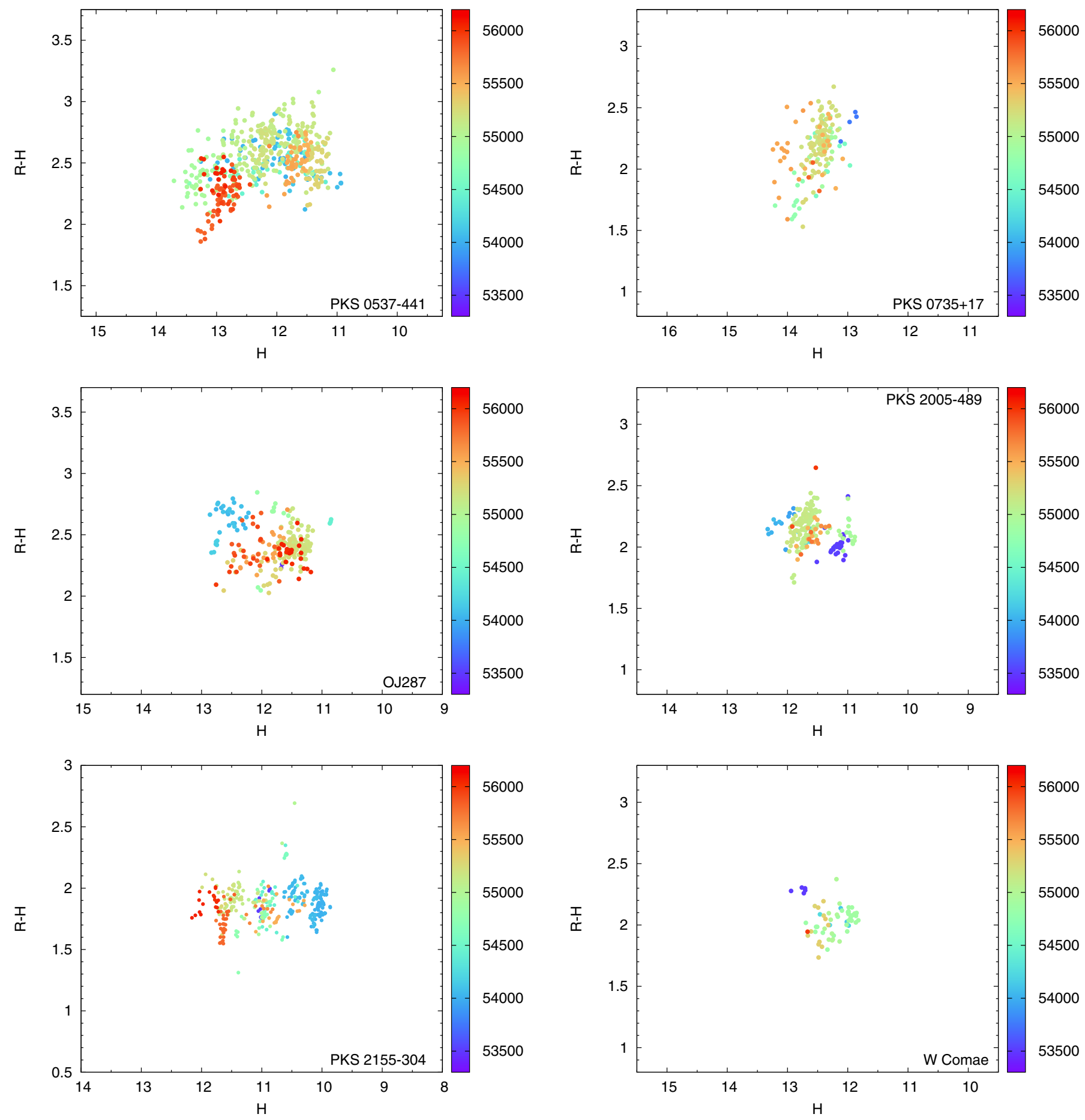

Fig. 6. Color-intensity diagrams for the BL Lacs. The color of each point is related to the epoch of detections of corresponding data, according to the bar on the right. Error bars are omitted for readability.

were performed within multiwavelength campaigns, where the optical and NIR monitoring was ancillary to X-ray or gamma ray $(\mathrm{GeV}, \mathrm{TeV})$ programs. In these cases the rate of monitoring could be typically once a day, or even once a week. The data suitable to search for variability on a time scale of hours are limited to a few epochs, while on much shorter time scales, the constraint is on the poor count statistics, owing to the diameter of the REM mirror. The threshold on the minimum flux detectable with a given integration time also depends on the mirror reflectance status, which has worsened after 2009, until a general maintenance intervention in 2012. When we were searching for rapid episodes, in addition to the visual control of each frame (Sect. 2), we chose to consider only those states over a minimum flux $f_{\text {min }} \sim 3 \mathrm{mJy}$ in the optical bands, and $\simeq 1 \mathrm{mJy}$ in the NIR bands. For fluxes $<f_{\min }$, no search of rapid variability events was performed. To identify potentially relevant variability events, we followed a procedure analogous to the one described by Montagni et al. (2006). We considered a series of $N$ consecutive observations, 
A. Sandrinelli et al.: Variability of seven blazars in six bands
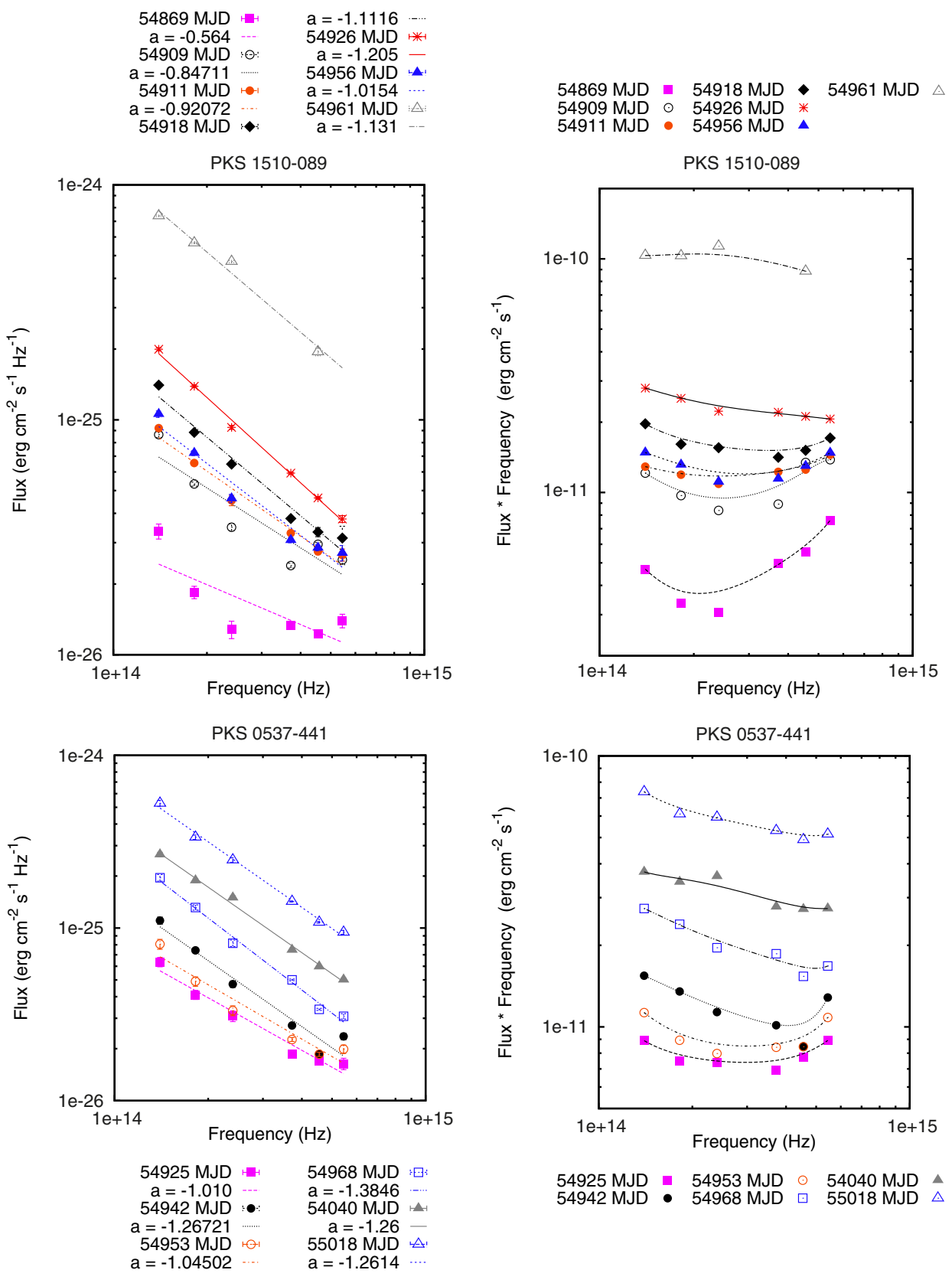

54925 MJD - 54953 MJD $\odot 54040$ MJD $\Delta$ 54942 MJD • 54968 MJD $\square 55018$ MJD $\triangle$
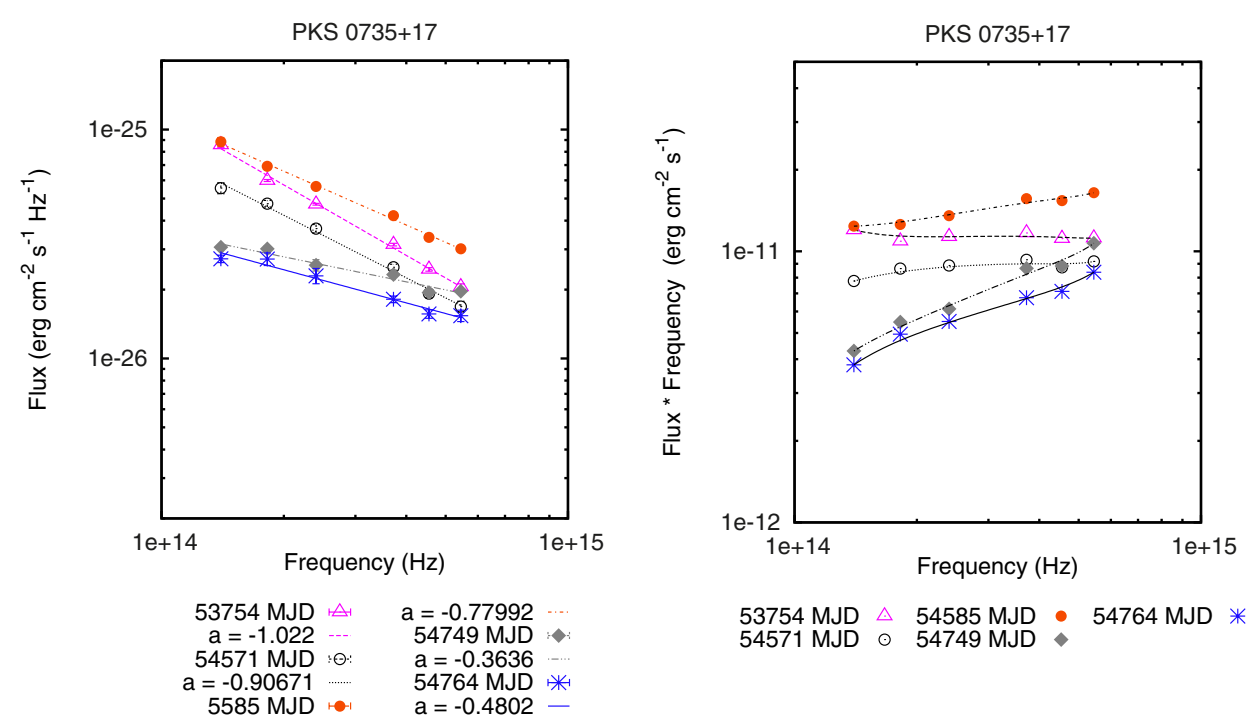

53754 MJD $\triangle 54585$ MJD -54764 MJD * 54571 MJD $\odot 54749$ MJD

Fig. 7. Spectral flux distribution (left pan$e l s$ ) and spectral energy distribution (right panels). The lines in the left panels are power-law fits and corresponding slopes $a$ are reported. In the right panels the lines are a help for the eye. 

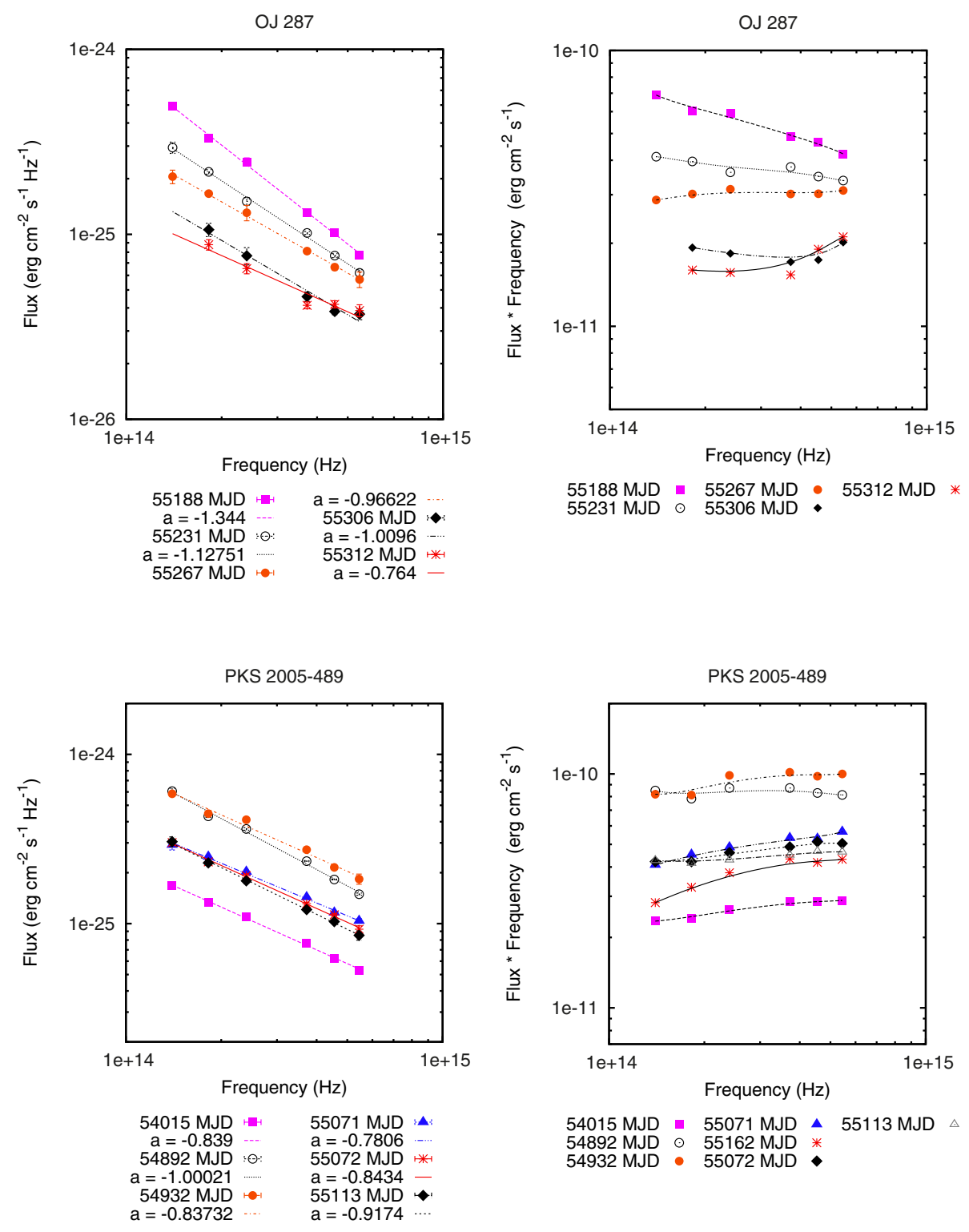

54015 MJD - 55071 MJD A 55113 MJD

54892 MJD $\odot 55162$ MJD *

54932 MJD $\bullet 55072$ MJD

Fig. 7. continued.

for each of which the flux is measured. The flux-time dependence is best fitted with a linear relation, which yields a value of $\mathrm{d} f / \mathrm{d} t$. A time scale is defined as

$$
\tau=\frac{\langle f\rangle}{\mathrm{d} f / \mathrm{d} t} \frac{1}{(1+z)}
$$

where $\langle f\rangle$ is the average value of the flux and $z$ the redshift. The search process for rapid events is fully automatized. An "event" is selected when $\tau$ is less than a given $\tau_{0}$, and the uncertainty on $\tau$ is less than $\tau / 2$. The event is discarded if the check star is significantly variable, and whenever $\tau$ is equal to or larger than $\xi^{-1} \tau_{\mathrm{ck}}$, where $\tau_{\mathrm{ck}}$ is the variability time scale of the check star, and $\xi$ is a fixed value. All the images of an event are then visually examined, excluding the cases where the source or the reference stars are near the image borders or cases where spurious tracks or spots are apparent.

First we looked for relatively long and well documented events, considering $N \geqslant 30$ in the same night, with a value of $\tau<12 \mathrm{~h}$ and $\xi=10$. We have retrieved two events of this type. The one referring to PKS 0537-441 (53 619 MJD) is reported in Fig. 8, see also Table 8 . The linear time scale is $\sim 12 \mathrm{~h}$.
Following the procedure suggested by Abdo et al. (2010) for Fermi gamma rays sources and adopted by Danforth et al. (2013) for UV photometry of the BL Lac object S5 0716+714, we also fitted the points in the night of the event with the function

$f(t)=f_{0}+a\left(\mathrm{e}^{(b-t) / \tau_{\mathrm{r}}}+\mathrm{e}^{(t-b) / \tau_{\mathrm{d}}}\right)^{-1}$

where $f_{0}$ is a constant underlying flux level, $a$ is a measure of the amplitude of the event, $b$ roughly describes the time of the peak of the event, and $\tau$ parameters are the rise and decay time scales. The best fit is given in Fig. 8, and the relevant time scales are $\tau_{\mathrm{r}} \sim$ $1.5 \mathrm{~h}$ and $\tau_{\mathrm{d}} \sim 12 \mathrm{~min}$ (see Table 8). Unfortunately during the night of the event, only $V$ photometry was collected. The event has some similarity with a variability episode of PKS 0537-441 deriving from the analysis of the REM archives, which is reported by Impiombato et al. (2011) and which was discussed in some detail by Zhang et al. (2013). However a careful analysis of the CCD images has shown the presence of a dark spot on the camera focal plane making the reliability of the event dubious, which therefore here is ignored.

The second event refers to PKS 2155-304 (53678 MJD), see Fig. 8 and Table 8 . The event was discovered with the automatic procedure in the $V$ band, and has a very similar counterpart in 

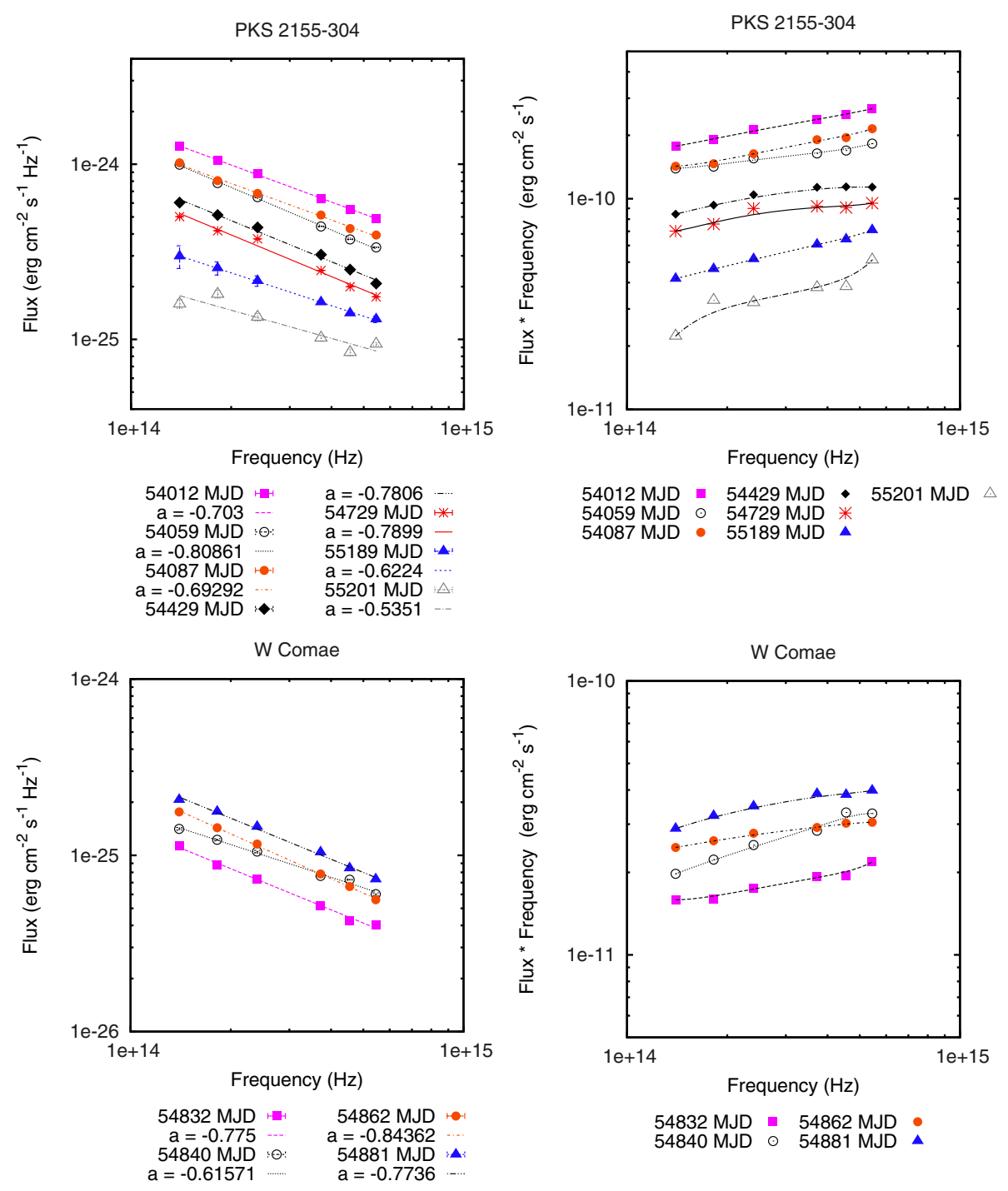

Table 8. Rapid event time scales.

\begin{tabular}{|c|c|c|c|c|c|c|c|c|}
\hline \multirow[t]{2}{*}{ Source } & \multirow{2}{*}{ Filter } & \multirow{2}{*}{ MJD } & \multirow[t]{2}{*}{ 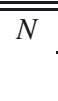 } & \multicolumn{2}{|r|}{ Linear fitting } & \multicolumn{3}{|c|}{ Exponential fitting } \\
\hline & & & & $\tau$ & $\tau_{\mathrm{ck}}$ & $\tau_{\mathrm{r}}$ & $\tau_{\mathrm{d}}$ & reduced $\chi^{2}$ \\
\hline PKS 0537-441 & V & 53619 & 198 & $11 \mathrm{~h} 53 \mathrm{~min} \pm 16 \mathrm{~min}$ & $34 \mathrm{~d} 17 \mathrm{~h} 22 \mathrm{~min} \pm 1 \mathrm{~h} 56 \mathrm{~min}$ & $1 \mathrm{~h} 27 \mathrm{~min} \pm 6 \mathrm{~h} 48 \mathrm{~min}$ & $12 \min 24 \mathrm{~s} \pm 8 \min 13 \mathrm{~s}$ & 1.13 \\
\hline \multirow[t]{4}{*}{ PKS 2155-304 } & $H$ & 55837 & 10 & $2 \mathrm{~h} 15 \mathrm{~min} \pm 22 \mathrm{~min}$ & $1 \mathrm{~d} 7 \mathrm{~h} 3 \mathrm{~min} \pm 22 \mathrm{~h} 16 \mathrm{~min}$ & & $22 \mathrm{~min} \pm 1 \mathrm{~h} 30 \mathrm{~min}$ & 4.60 \\
\hline & $R$ & 53678 & 25 & $11 \mathrm{~h} 54 \mathrm{~min} \pm 25 \mathrm{~min}$ & $6 \mathrm{~d} 10 \mathrm{~h} 34 \mathrm{~min} \pm 1 \mathrm{~d} 23 \mathrm{~h} 48 \mathrm{~min}$ & & $1 \mathrm{~h} 21 \mathrm{~min} \pm 39 \mathrm{~min}$ & 5.78 \\
\hline & $V$ & 53678 & 31 & $12 \mathrm{~h} 00 \mathrm{~min} \pm 21 \mathrm{~min}$ & $7 \mathrm{~d} 16 \mathrm{~h} 28 \mathrm{~min} \pm 3 \mathrm{~d} 17 \mathrm{~h} 44 \mathrm{~min}$ & & $1 \mathrm{~h} 39 \mathrm{~m} \pm 39 \mathrm{~min}$ & 1.16 \\
\hline & V & 53973 & 10 & $2 \mathrm{~h} 15 \mathrm{~min} \pm 14 \mathrm{~min}$ & $3 \mathrm{~d} 17 \mathrm{~h} 33 \mathrm{~min} 25 \mathrm{~s} \pm 1 \mathrm{~d} 11 \mathrm{~h} 40 \mathrm{~min}$ & & $3 \min 14 \mathrm{~s} \pm 2 \min 1 \mathrm{~s}$ & 1.79 \\
\hline
\end{tabular}

Notes. The exponential fits refer to Eq. (3). For the episodes related to PKS 2155-304, in Eq. (3) $\tau_{\mathrm{r}}$ is assumed equal to $\tau_{\mathrm{d}}$.

the $R$ band. No rapid event was detected in NIR bands where coverage is poorer. The continuous lines in Fig. 8 are the fits with exponentials following Abdo et al. (2010).

We then looked for more rapid events. We have fixed a time scale $\tau_{0}<3 h, \xi=4$ and a number $N=8$ of points. The only two events that we retrieved both refer to PKS 2155-304, see Fig. 9 and Table 8. The exponential time scales are now of the order of minutes. The event occurred on 53973 MJD yields a time scale of three minutes and the reduced $\chi^{2}$ indicates an acceptable fit with an exponential function. The case in 55837 MJD is more complex. It seems that, superimposed on the exponential rise, there may be a spike that is, however, represented by a single point. We detect for both these episodes the very fast variability rate of $0.43 \mathrm{mag} / \mathrm{h}$, comparable to the variability rate of $0.45 \mathrm{mag} / \mathrm{h}$ found by Danforth et al. (2013) for S5 0716+714.

\section{Discussion}

For the sources where the SED of the lower states are not well fitted by a power law (PKS 1510-089, PKS 0537-441, OJ 287, see Fig. 7), the indication is that the NIR bands yield a powerlaw index, which is unchanged with respect to the high states obtained by combining NIR and optical observations. In the lowest states a new component becomes apparent that shows up in the 

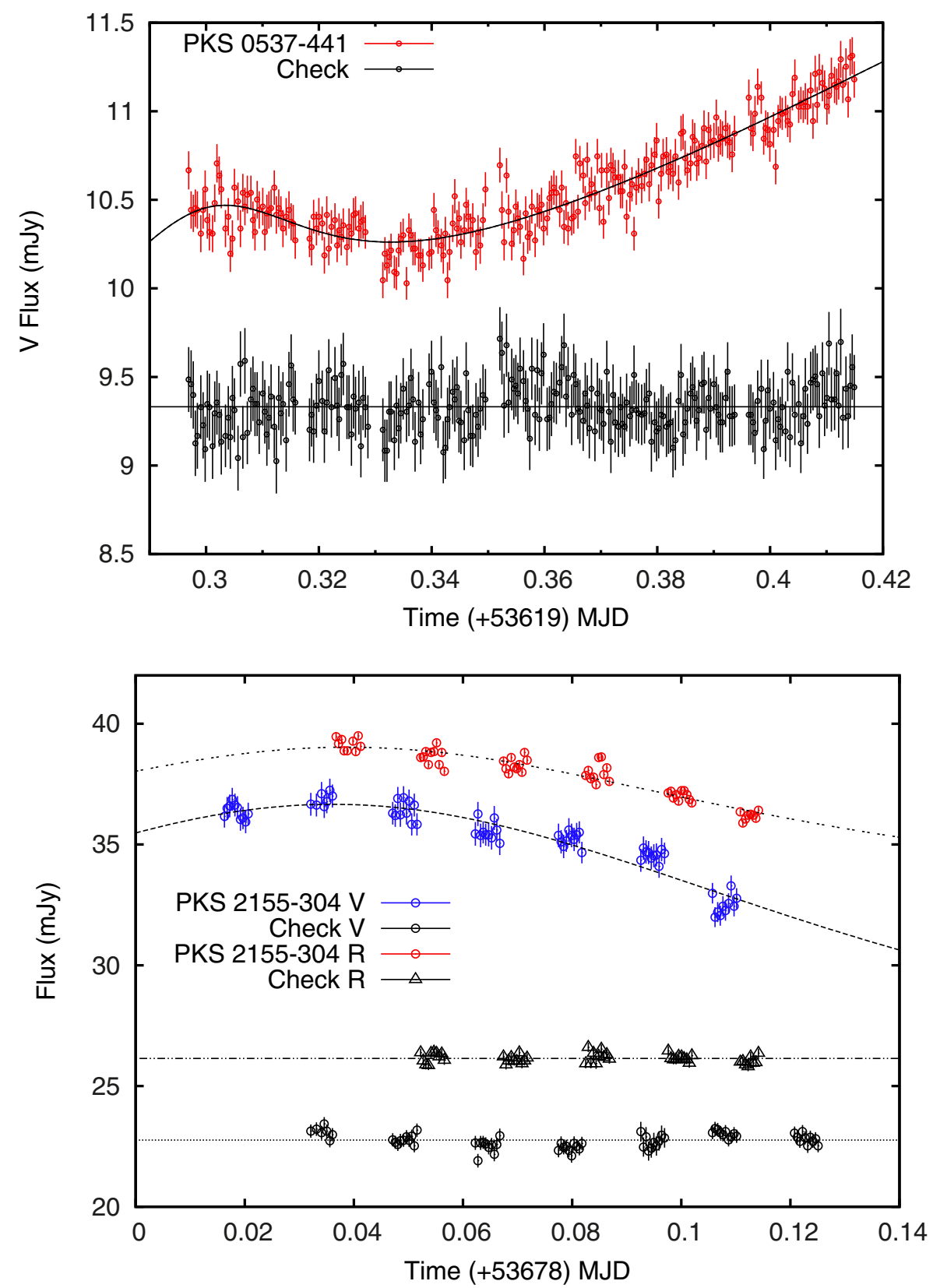

Fig. 8. Episodes of intra-night variability in PKS 0537-441and in PKS 2155-304. The check data has been multiplied $\times 3$ for PKS $2155-304$ and $\times 2$ for PKS 0537-441.The lines are the best fits using Eq. (3). optical. This component could be thermal, but this cannot be assessed, owing to the limited bandwidth covered by our six filters. In any case, the second component appears to be bluer than the power law. If the power law represents the variable part, this is consistent with the behavior of the variability index $\sigma_{\text {rms }}^{2}$, which tends to be larger in the NIR with respect to the optical bands (see Table 6).

As shown in the previous section we have selected a number of episodes with time scales of hours or even shorter. Our selection criteria have been rather stringent. There is a comparable number of events, which may be real but do not satisfy one of our criteria. All the events derive from two sources that are very variable on time scales longer than one day, i.e. PKS 0537-441 and PKS 2155-304, and for which the total coverage is the largest, even with regard to the intra-night monitoring programs. For the other very variable source PKS 1510-089, we collected a number of a priori interesting events, but they corresponded to very low states of the source, and therefore are not considered here for the criterion given at the beginning of Sect. 5. Even when also taking the unconfirmed rapid events into account, one finds that hour time scales are rare. The ratio of their summed duration to the total exposure time is $\sim 8 / 670$ for PKS $0537-441$ and $\sim 20 / 730$ for PKS 2155-304, where $670 \mathrm{~h}$ and $730 \mathrm{~h}$ are the overall observing times of the two sources. The discovery of these rapid events therefore requires very long monitoring campaigns. This inference cannot be applied to the case of S5 $0716+714$, where the one and only COS-HST observation found a rapid event.

The two most extreme events are those referring to PKS 2155-304 reported in Fig. 9, where the time scale events for a linear fit are on the order of hours, and those for the exponential fit are a few minutes. Similar short time scales have been found at $\lambda=1400 \AA$ by Danforth et al. (2013) during the above-mentioned observation of S5 0716+714. In PKS 2155304 sub-hour time scales have appeared in the $\mathrm{TeV}$ band during the famous active state on July 29-30, 2006 (Aharonian et al. 2007). The mass of the black hole in PKS 2155-304, is most probably $\sim 10^{9} M_{\odot}$ (Falomo et al. 1991; Kotilainen et al. 1998), 


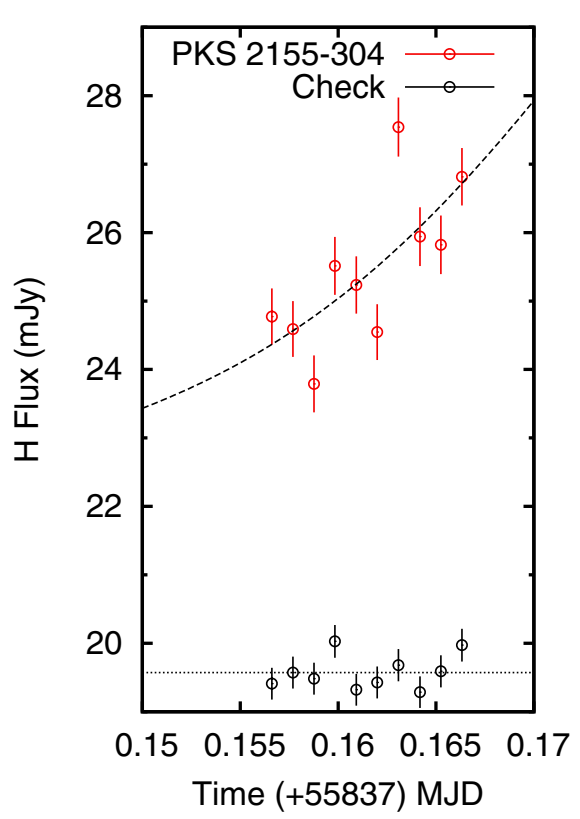

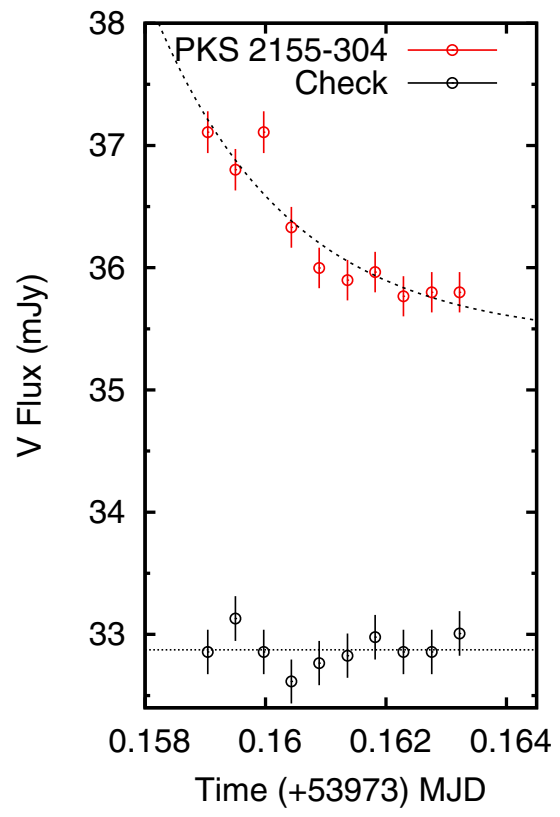

Fig. 9. Short variability events. In the right panel the check data has been divided by 2.5 . In the left panel the check data has been multiplied $\times 1.4$. The lines are the best fits using Eq. (3). corresponding to a Schwarzschild radius $r_{\mathrm{G}} \sim 2 \times 10^{14} \mathrm{~cm}$. Assuming an emitting region of a few (say 5) $r_{\mathrm{G}}$, the corresponding time scales are

$\tau \sim \frac{R(1+z)}{c \delta}=\frac{4 \times 10^{4} \mathrm{~s}}{\delta}$

where $\delta$ is the Doppler factor. A direct comparison with the reported time scales would require a specific model of the source, indicating in particular if the time scale to be considered is from the linear or exponential fit. In any case the reported rapid optical episodes indicate that the requirement of large Doppler factors is inevitable, in agreement with what is concluded for the variability at high energies.

Acknowledgements. We are grateful to the referee for a very positive and constructive report. We also thank Dino Fugazza for his efficient management of the REM observations and archive. This work has been supported by ASI grant I/004/11/0 and by PRIN-MIUR 2009 grants.

\section{References}

Abdo, A. A., Ackermann, M., Ajello, M., et al. 2010, ApJ, 722, 520

Aharonian, F., Akhperjanian, A. G., Bazer-Bachi, A. R., et al. 2007, ApJ, 664, L71

Blandford, R. D., \& Rees, M. J. 1978, Phys. Scr., 17, 265

Bonning, E., Urry, C. M., Bailyn, C., et al. 2012, ApJ, 756, 13

Cardelli, J. A., Clayton, G. C., \& Mathis, J. S. 1989, ApJ, 345, 245

Chatterjee, R., Fossati, G., Urry, C. M., et al. 2013, 2012 Fermi Symp. Proc., eConFC121028 [arXiv: 1303.2095]

Ciprini, S., Takalo, L. O., Tosti, G., et al. 2007, A\&A, 467, 465

Conconi, P., Cunniffe, R., D’Alessio, F., et al. 2004, Proc. SPIE, 5492, 1602

Covino, S., Stefanon, M., Sciuto, G., et al. 2004, Proc. SPIE, 5492, 1613

Covino, S., L., Treves, A., \& Sbarufatti, B. 2010, 38th COSPAR Scientific Assembly, 38, 5

D'Ammando, F., Raiteri, C. M., Villata, M., et al. 2010a, 38th COSPAR Scientific Assembly, 38, 7

D'Ammando, F., Raiteri, C. M., Villata, M., et al. 2010b, in Accretion and Ejection in AGN: a Global View, eds. L. Maraschi, G., Ghisellini, \& R. Della Ceca, ASP Conf. Ser., 427, 271
D’Ammando, F., Vercellone, S., Donnarumma, I., et al. 2010c, X-ray Astronomy 2009; Present Status, Multi-Wavelength Approach and Future Perspectives, AIP Conf. Ser., 1248, 415

D’Ammando, F., Bulgarelli, A., Chen, A. W., et al. 2011a, Adv. Space Res., 48, 76

D’Ammando, F., Vercellone, S., Donnarumma, I., et al. 2011b, J. Phys. Conf. Ser., 280, 012002

D'Ammando, F., Raiteri, C. M., Villata, M., et al. 2011c, A\&A, 529, A145

D'Ammando, F., Antolini, E., Tosti, G., et al. 2013, MNRAS, 431, 2481

Danforth, C. W., Nalewajko, K., France, K., \& Keeney, B. A. 2013, ApJ, 764, 57

Dolcini, A., Covino, S., Treves, A., et al. 2005, A\&A, 443, L33

Dolcini, A., Farfanelli, F., Ciprini, S., et al. 2007, A\&A, 469, 503

Edelson, R., Turner, T. J., Pounds, K., et al. 2002, ApJ, 568, 610

Edelson, R., Mushotzky, R., Vaughan, S., et al. 2013, ApJ, 766, 16

Falomo, R., Giraud, E., Melnick, J., et al. 1991, ApJ, 380, L67

Fiorucci, M., \& Tosti, G. 1996, A\&AS, 116, 403

Foschini, L., Treves, A., Tavecchio, F., et al. 2008, A\&A, 484, L35

González-Pérez, J. N., Kidger, M. R., \& Martín-Luis, F. 2001, AJ, 122, 2055

Hamuy, M., \& Maza, J. 1989, AJ, 97, 720

Impiombato, D., Tosti, G., Treves, A., et al. 2008, in Proc. Blazar Variability across the Electromagnetic Spectrum, 43, available at http://pos.sissa. it

Impiombato, D., Covino, S., Treves, A., et al. 2011, ApJS, 192, 12

Kotilainen, J. K., Falomo, R., \& Scarpa, R. 1998, A\&A, 336, 479

Massaro, E., Giommi, P., Leto, C., et al. 2012, Multifrequency Catalogue of Blazars, 3rd edn. (Rome, Italy: ARACNE editrice)

Montagni, F., Maselli, A., Massaro, E., et al. 2006, A\&A, 451, 435

Nandra, K., George, I. M., Mushotzky, R. F., Turner, T. J., \& Yaqoob, T. 1997, ApJ, 476, 70

Pian, E., Romano, P., Treves, A., et al. 2007, ApJ, 664, 106

Pucella, G., D'Ammando, F., Romano, P., et al. 2010, A\&A, 522, A109

Rector, T. A., \& Perlman, E. S. 2003, AJ, 126, 47

Sasada, M., Uemura, M., Fukazawa, Y., et al. 2011, PASJ, 63, 489

Schlegel, D. J., Finkbeiner, D. P., \& Davis, M. 1998, ApJ, 500, 525

Skrutskie, M. F., Cutri, R. M., Stiening, R., et al. 2006, AJ, 131, 1163

Tosti, G., Bagaglia, M., Campeggi, C., et al. 2004, Proc. SPIE, 5492, 689

Vaughan, S., Edelson, R., Warwick, R. S., \& Uttley, P. 2003, MNRAS, 345, 1271

Zerbi, F. M., \& Rem Team 2001, Astron. Gesellschaft Meeting Abstracts, 18, JD 0101

Zhang, B.-K., Wang, S., Zhao, X.-Y., Dai, B.-Z., \& Zha, M. 2013, MNRAS, 428, 3630 